Review

\title{
Chitosan Nanoparticles-Based Ionic Gelation Method: A Promising Candidate for Plant Disease Management
}

\author{
Nguyen Huy Hoang ${ }^{1}{ }^{(0)}$, Toan Le Thanh ${ }^{2}$, Rungthip Sangpueak ${ }^{1}$, Jongjit Treekoon ${ }^{3}$, Chanon Saengchan ${ }^{1}$, \\ Wannaporn Thepbandit ${ }^{1}\left(\mathbb{D}\right.$, Narendra Kumar Papathoti ${ }^{1}{ }^{\circledR}$, Anyanee Kamkaew ${ }^{3}$ and Natthiya Buensanteai ${ }^{1, *}$
}

1 School of Crop Production Technology, Institute of Agricultural Technology, Suranaree University of Technology, Nakhon Ratchasima 30000, Thailand; huyhoangqct@gmail.com (N.H.H.); fongfangfang_m5430222@hotmail.com (R.S.); c.saengchan5310@gmail.com (C.S.); w.thepbandit@gmail.com (W.T.); narendrakumar.papathoti@gmail.com (N.K.P.)

2 Department of Plant Protection, College of Agriculture, Can Tho University, Can Tho 900000, Vietnam; 1ttoan@ctu.edu.vn

3 School of Chemistry, Institute of Science, Suranaree University of Technology, Nakhon Ratchasima 30000, Thailand; yuiyongyuy@gmail.com (J.T.); anyanee@g.sut.ac.th (A.K.)

* Correspondence: natthiya@sut.ac.th

check for updates

Citation: Hoang, N.H.; Le Thanh, T.; Sangpueak, R.; Treekoon, J.; Saengchan, C.; Thepbandit, W.; Papathoti, N.K.; Kamkaew, A.; Buensanteai, N. Chitosan Nanoparticles-Based Ionic Gelation Method: A Promising Candidate for Plant Disease Management. Polymers 2022, 14, 662. https://doi.org/ $10.3390 /$ polym 14040662

Academic Editor: Luminita Marin

Received: 13 January 2022

Accepted: 2 February 2022

Published: 9 February 2022

Publisher's Note: MDPI stays neutral with regard to jurisdictional claims in published maps and institutional affiliations.

Copyright: (C) 2022 by the authors. Licensee MDPI, Basel, Switzerland. This article is an open access article distributed under the terms and conditions of the Creative Commons Attribution (CC BY) license (https:// creativecommons.org/licenses/by/ $4.0 /$ )

\begin{abstract}
By 2050, population growth and climate change will lead to increased demand for food and water. Nanoparticles (NPs), an advanced technology, can be applied to many areas of agriculture, including crop protection and growth enhancement, to build sustainable agricultural production. Ionic gelation method is a synthesis of microparticles or NPs, based on an electrostatic interaction between opposite charge types that contains at least one polymer under mechanical stirring conditions. NPs, which are commonly based on chitosan (CS), have been applied to many agricultural fields, including nanopesticides, nanofertilizers, and nanoherbicides. The CS-NP or CS-NPs-loaded active ingredients ( $\mathrm{Cu}$, saponin, harpin, $\mathrm{Zn}$, hexaconazole, salicylic acid (SA), NPK, thiamine, silicon, and silver (Ag)) are effective in controlling plant diseases and enhancing plant growth, depending on the concentration and application method by direct and indirect mechanisms, and have attracted much attention in the last five years. Many crops have been evaluated in in vivo or in greenhouse conditions but only maize (CS-NP-loaded Cu, Zn, SA, and silicon) and soybean (CS-NP-loaded Cu) were tested for manage post flowering stalk rot, Curvularia leaf spot, and bacterial pustule disease in field condition. Since 2019, five of eight studies have been performed in field conditions that have shown interest in CS-NPs synthesized by the ionic gelation method. In this review, we summarized the current state of research and provided a forward-looking view of the use of CS-NPs in plant disease management.
\end{abstract}

Keywords: active ingredient; chitosan; ionic gelation method; nanoparticle; plant disease management

\section{Introduction}

The world population is predicted to reach 9.8 billion by 2050. Demand for food and water will increase, especially in developing countries, where incomes are projected to increase dramatically [1]. Over the period 2010-2050, the total food demand is projected to increase by 35 to $56 \%$ and the population at risk of hunger to change from -91 to $+8 \%$. Food production is annually affected by climate change as well as pest and disease damage. When affected by climate change, the total food demand and the population at risk of hunger increases, namely, from 30 to $62 \%$ and -91 to $+30 \%$ [2]. Moreover, food production is severely affected by pests and phytopathogens. Plant diseases caused a $16 \%$ loss in global crop production between 2001 and 2003 [3]. According to an assessment of [4], phytopathogens cause a $25 \%$ yield loss in developing countries. Among them, fungi are the most common $(42 \%)$, followed by bacteria $(27 \%)$, viruses $(18 \%)$, and nematodes $(13 \%)$. Phytopathogens cause both direct and indirect effects. The consequences of harmful effects 
are not only loss of productivity; more seriously, but also food quality and toxin production (Aspergillus, Fusarium) [5]. In severe cases, the potato late blight (Phytophthora infestans) break-out caused the historic Irish famine in the mid-19th century [3]. In the research model of [6], when blast rice disease (Magnaporthe oryzae) occurred seriously in one country (Thailand) to Southeast Asia, that significantly increased the global rice price, especially for the rice importing countries in sub-Saharan Africa. Disease control or management is essential. Most farmers in developing countries (Laos, Cambodia, and Vietnam) are aware of the risks of pesticides, but they often consider pesticides as an indispensable tool in pest control [7]. A review of pesticide efficacy on controlling arthropod pests, crop pathogens, and weeds indicated that approximately $0.1 \%$ of pesticides applied reach their target organism, and pesticides directly affect the health of farmers and pollute the environment [8]. The number of pesticides retained on crops depends on many factors, including the formulation of pesticide used, the volume of spray applied, the type of equipment used, and the quality of the spray [9]. The harmful effect of pesticides on the environment and human health demands research on new harmless means of disease control, to satisfy food security for world's population. Increasing sustainable agricultural production, reducing food waste, and applying new technology are proposed to ensure food security [10].

In recent years, nanotechnology has emerged as a trend in agricultural production. Nanoparticles (NPs) are very small but powerful and can be applied in many agricultural fields. Nanofertilizers and nanobiotechnology can be used in enhancing crop yields by providing absorbable nutrients and genetic materials, respectively. Water purification, packaging, plant growth, and seed germination were also improved. Furthermore, nanomaterials can be applied to the soil or supplied directly to crops to increase soil health or crop health against adverse environmental factors such as drought, salinity, UV-B, heat, heavy mental, flooding, and biotic stress. Additionally, highly sensitive and precise nanosensors have been used in prediction to make precise farming decisions. Moreover, nanopesticides (nanofungicides, nanobactericides, and nanoinsecticide), nanoelicitors, and nanoherbicides have been applied to protect plants from arthropod pests, phytopathogens, and weeds [11-17]. Polymers such as gum, mucilage, and chitosan (CS) are naturally derived, readily available, inexpensive, convertible, and biodegrable. Drug-delivery-system-based natural polymers are promising candidates that can be applied in pharmaceutical, biomedical, and agricultural production, with advantages including nontoxicity, water solubility, biocompatibility, and multiple function [18-22]. In the field of plant protection, NPs can be applied as protectors or carriers to load active ingredients to protect plants against insects, fungi, bacteria, and viruses [23]. With their small size, positive charge, and large surface area, NPs have high reactivity and easily penetrate cells through foliage, brand, trunk, root or stick on plant parts [24,25]. According to the review of [26], micronutrient NPs $(\mathrm{Cu}$, $\mathrm{ZnO}, \mathrm{MgO}$, and $\mathrm{ZnO}$ ) and non-micronutrient NPs (Ag) could improve plant growth and inhibit plant pathogens such as Phoma destructive, P. infestans, Rhizopus stolonifer, Mucor plumbeus, Fusarium oxysporum, Botrytis cinerea, P. cubensis, P. syringae pv. lachrymans, and Colletotrichum spp. In addition, when using NPs as carriers, they can control the released active ingredients to increase uptake, availability of water, and nutrients and reduce the negative environmental effect. Nanopesticides have a high shelf life, site-specific uptake, solubility, low soil leaching, and toxicity $[23,27]$, increasing the efficacy of NPs in controlling plant diseases and enhancing plant growth.

There are two approaches to synthesize NPs, including top-down and bottom-up methods. The physical, chemical, and biological methods can all be used to synthesize NPs and require different equipment and materials, leading to produced NP particles having different characteristics, properties, and functions [28,29]. The ionic gelation technique is a chemical method to synthesize microparticles or NPs based on electrostatic interactions between ions with different charges that was discovered by Calvo et al. (1997) [30,31]. This technique requires polymeric, usually CS and alginate. The cation of CS $\left(\mathrm{R}_{-} \mathrm{NH}_{3}{ }^{+}\right)$ crosslink with polyanion of sodium triphosphate (TPP) (phosphoric ion) under constant 
stirring conditions leads to hydrogel formation. The reaction efficiency or properties of the NPs (size, polydispersity indexes determined) differ depending on the ratio of CS and TPP. The process consists of three phases which are solution, aggregation, and opalescent suspension. The materials and instruments for this method can be easily found in conventional laboratories [32-37]. Previously, this CS-NPs method was attended in pharmacy to control bacteria on people. The authors of [32] have synthesized CSNPs-loaded with various metals, including $\mathrm{Ag}, \mathrm{Cu}, \mathrm{Zn}, \mathrm{Mn}$, or Fe, by ionic gelation method. Antimicrobial activity test showed that NPs except Fe could inhibit Escherichia coli, Salmonella choleraesuis, and Staphylococcus aureus at low concentrations, from 3-85 $\mu \mathrm{g} / \mathrm{mL}$, among them, the minimum inhibitory concentration of CS-NPs-loaded Ag and Cu from 3-9 and 9-21 $\mu \mathrm{g} / \mathrm{mL}$, respectively. In addition, the study of [38] showed that CS-NP loaded Ag could inhibit $S$. aureus, E. coli, and Klebsiella pneumonia with minimum concentrations of $1.69,1.69$, and $3.38 \mu \mathrm{g} \mathrm{Ag} / \mathrm{mL}$, respectively. Moreover, there have been many studies applying NPs synthesized by this method to control plant diseases and enhancing plant growth, most of which are related to CS (shown below). The present article provides a general review of CS-NPs synthesized by the ionic gelation method and their application in plant disease management.

\section{Ionic Gelation Method}

In 1997, two publications by Calvo et al. about the synthesis of NPs by a method called ionic (ionotropic) gelation. There, TPP solution is added to CS and/or diblock copolymer of ethylene oxide and propylene oxide under stirring conditions, leading to the formation of CS-NP particles, with a size 200-1000 nm and zeta potential of 20-60 mV, depending on mass ratio CS/TPP or molecular weight of CS. This hydrogel formation is known due to the electrostatic interactions of the amino group of CS and the polyanion group of TPP. In addition, in these studies, bovine serum albumin (protein), tetanus, and diphtheria toxoid (vaccine) were also successfully loaded into NPs [30,31]. Since then (2021), approximately 11,700 research and review articles related to this method have been published according to the statistics of Google Scholar (Figure 1) [39]. Interestingly, the number of articles has increased year by year, reaching a peak in 2021 with 2090 publications. This shows the researchers' interest in the ionic gelation method and NPs.

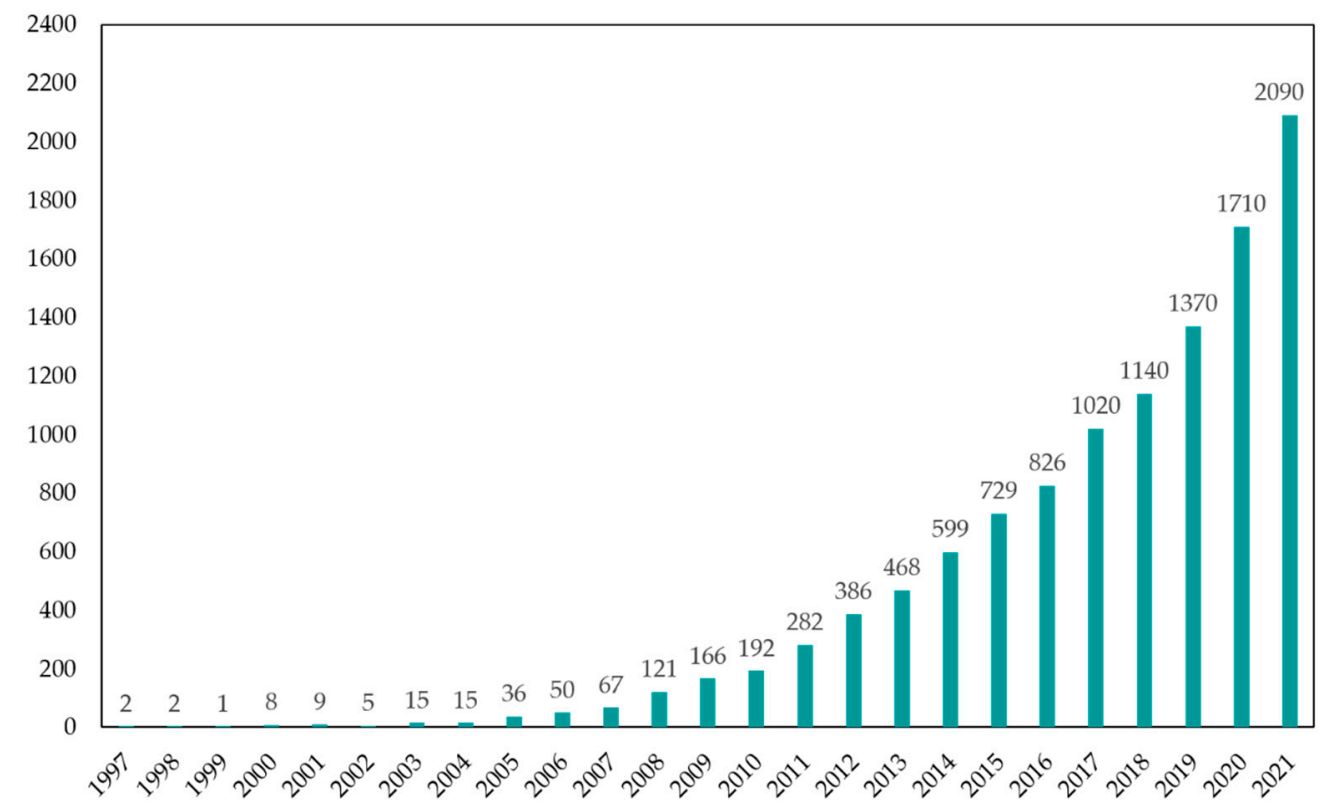

Figure 1. The number of articles in Google Scholar searched by key word "ionic gelation" + "nanoparticles" [39]. 
In general, this is a method to form microparticles or NPs which based on electrostatic interaction between opposite charge types that contain at least one polymer under mechanical stirring conditions [37]. On the records of [37,40,41], polymers including CS, carboxymethyl cellulose, collagen, dextran, fibrin, gelatin, gellan gum, hyaluronic acid, sodium alginate, pectin, and anions, including chloride salts $(\mathrm{Ba}, \mathrm{Ca}, \mathrm{Mg}, \mathrm{Cu}, \mathrm{Zn}$, and $\mathrm{Co})$, sulfate salts ( $\mathrm{Na}, \mathrm{Mg}$, or octyl-, lauryl-, hexadecyl-, cetostearyl-), polyphosphate salts (pyro-, tri-, tetra-, octa-, and hexameta-), ferrocyanide, and ferricyanide salts were used for synthesis of NPs. Among them, CS (polymer-cation) and TPP (anion) are most commonly used. Furthermore, drugs or bioactive molecules can be encapsulated into matrix of NPs to increase their efficacy.

CS is a natural polysaccharide, produced by the alkaline deacetylation of chitin, which possesses excellent characteristics, including low toxicity, low cost, biodegradability, biocompatibility, environmental non-toxicity, and adsorption abilities [18,42,43]. With its superiority, CS is used in wastewater treatment, cosmetics, toiletries, food, beverages, agrochemicals, and pharmaceuticals, and its production in the South-East Asian region reaches 1.5 million tons per year $[44,45]$. The properties of CS can be modified by chemical and/or mechanical processes by hydroxide and/or amide groups, respectively [46]. TPP is also a safe material, commonly used in the synthesis of NPs by the ionic gelation method as a crosslinking agent $[37,47]$.

CS and TPP can be seen as a "legendary" pair of counter ions in the ionic gelation method because of their popularity in studies. Typically, cations and polyanions are released from dissolving CS and TPP in acetic acid and distilled water, respectively. When TPP is dripped into a CS solution, the polyanion (negative charge) bonds to an amino group (positive charge) by electrostatic interaction, which causes CS to undergo a gel ionization process, leading to the formation of NPs that are usually collected by centrifuge $[18,48,49]$. The primary interactions in ionic crosslinking configuration are H-link and T-link. The $\mathrm{H}$-link is interaction of $\mathrm{O}^{-}$and $\mathrm{NH}_{3}{ }^{+}$in the same plane, while the T-link is interaction of nonbrinding oxygen atom and $\mathrm{NH}_{3}{ }^{+}$in different plane (Figure 2) [34,37]. The formation of NPs is influenced by CS concentration, CS molecular weight, CS/TPP ratio, drug or bioactive molecules concentration, $\mathrm{pH}$, stirring, and centrifuge (time, speed) [48].

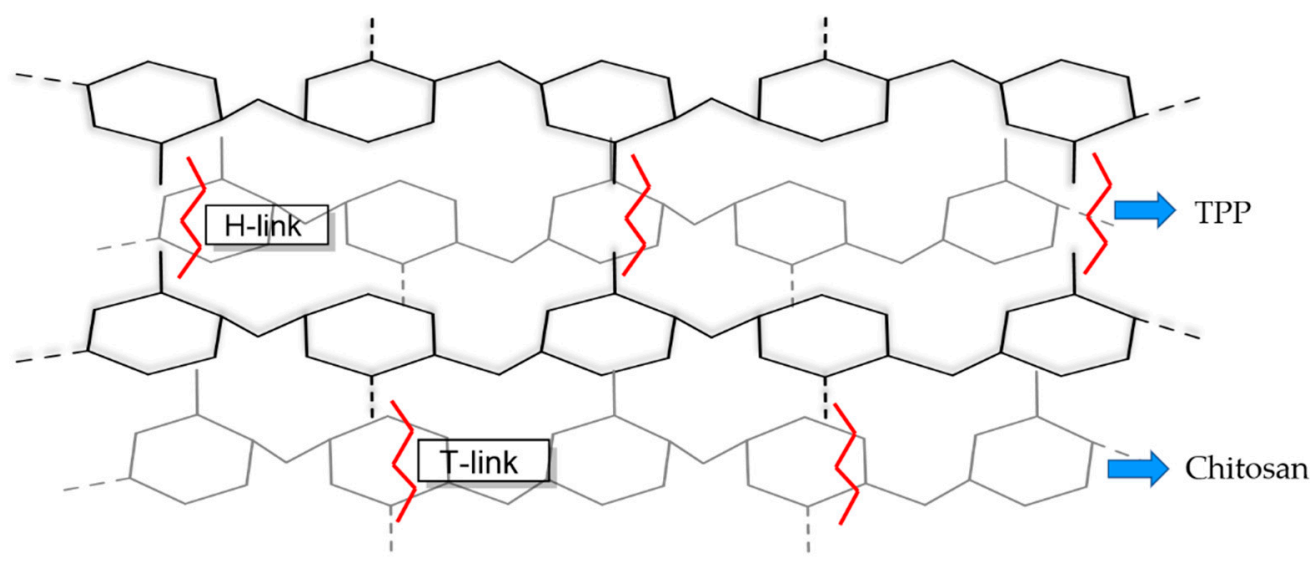

Figure 2. The electrostatic interactions between CS and TPP by an H-link configuration (in the same plane) and T-link configuration (in a different plane) in ionotropic gelation.

After the synthesis of NPs, their popular features include hydrodynamic diameter, zeta potential, polydispersity index (PDI), morphology, dry state diameter, interaction confirms, encapsulation efficiency (EE), loading capacity (LC), and crystal phase, defined by dynamic light scattering (DLS), scanning electron microscope (SEM), transmission electron microscopy (TEM), Fourier-transform infrared spectroscopy (FTIR), inductively coupled plasma atomic emission spectroscopy (ICP-OES), atomic absorption spectrometry 
(AAS), ultraviolet visible (UV-Vis), and X-ray diffraction techniques. These features will vary depending on the synthesis condition, see Tables 1 and 2 .

NPs include material with at least one dimension in 1-100 nanometers $(\mathrm{nm})$ range [50]. However, the size of the NPs synthesized by the ionic gelation method is usually greater than $100 \mathrm{~nm}$ [51-56]. The characteristics of CS-NPs synthesized according to the ionic gelation method are presented in Table 1. In the synthesis, CS-NPs, mass ratios, and volume ratios between CS and TPP vary from 1:10 to $15: 1$ and $1: 10$ to $25: 1[32,51,53,57]$. CS-NPs smaller than $100 \mathrm{~nm}$ are synthesized with CS and TPP mass-to-volume ratios reported in studies of [57] with 2:5 and 1:10 (50 nm), [58] with 5:4 and 1:1 $(2.3-7.5 \mathrm{~nm})$, [59] with 6:1 and 3:1 (83.32 nm), [60] with 5:1 and 5:1 (86.8 $\mathrm{nm})$, and [32] with 25:1 and 15:1 $(53.99 \mathrm{~nm})$. Similarly, CS-NPs larger than $100 \mathrm{~nm}$ were reported in studies of [52] with 3:1 (180.9-595.7 $\mathrm{nm})$, [51] with 1:10 and 1:1 (192.5 nm), [56] with 10:1 and 5:1 (126.2-167.1 nm), 8:1 and 4:1 (493.3-573.1 nm), [54] with 2:5 and $1: 1(100-1000 \mathrm{~nm})$, [53] with 5:1 and 2:1 $(238.17 \mathrm{~nm})$, and [55] with 5:1 and 1:2 (204.8-472.1 nm). CS molecular weight also influences NPs formation. As reported by [52], CS-NPs were synthesized from CS low molecular weight $(161 \mathrm{kDa})$ of $180.9 \mathrm{~nm}$ in size and were smaller than medium $(300 \mathrm{kDa})$ and high $(810 \mathrm{kDa})$ molecular weights of 309.9 and $339.4 \mathrm{~nm}$, respectively. The authors of [56] had various mass (10:1 and 8:1) and volume (5:1 and 4:1) ratios between CS and TPP with time stirring (60 and $30 \mathrm{~min}$ ). Results showed that CS-NPs with longer stirring would have smaller sizes in the same mass ratios of 126.2, 167.1, 493.3, and $573.1 \mathrm{~nm}$ for 10:1 and 5:1, 8:1, and 4:1 (volume ratio), respectively. This is slightly different from the study of [53] when mass ratio CS:TPP was increased from 5:1 to 20:1 with the same volume ratio, the NPs' size increased from 238.17 to $1315.37 \mathrm{~nm}$. The authors of [55] conducted research with various sonicate times for 3, 5, 10, and $20 \mathrm{~min}$, and the results showed that the NPs' size decreased (344.6, 472.1, 261.3, and $204.8 \mathrm{~nm}$, respectively). In addition to centrifuge, the authors of [52] adjusted the $\mathrm{pH}$ of the CS:TPP mixture to $4.5-5$ to collect NPs. The results showed that the NPs size was greater than that of the centrifugation method for low (180.9 and $225.7 \mathrm{~nm}$ ) and high (339.4 and $595.7 \mathrm{~nm}$ ) CS molecular weight and similar to medium molecular weight (309.9 and $301.5 \mathrm{~nm}$ ), respectively. The PDI of CS-NPs ranged from 0.195 [55] to 1.0 [32]. A low PDI shows a high uniform dispersion of the particles in the solution and vice versa [61]. CS-NPs have PDIs of 0.31-0.52 [52], 0.6 [51], 0.44-0.69 [56], and 0.195-0.57 [55]. Zeta potential is an effective electric charge on NPs' surface, from $-100 \mathrm{mV}$ to $100 \mathrm{mV}$, representing only NPs' stability [62]. Zeta potentials of CS-NPs are 21.7-45.6 $\mathrm{mV}$ [52], $45.3 \mathrm{mV}$ [51], $-28 \mathrm{mV}$ [59], $32.4 \mathrm{mV}$ [63], 20.8-27.8 $\mathrm{mV}$ [56], and $51.37 \mathrm{mV}$ [32]. CS-NPs were mostly spherical when observed under SEM or TEM [51,55,57-60,63], or sphere-like [54,56]. Furthermore, sizes under TEM (dry state) that were smaller than DLS (hydrodynamic size) were shown in the study of [58] with $1.5 \mathrm{~nm}$, [59] with 20-50 nm, [63] with 10-30 nm, and [54] with $100 \mathrm{~nm}$, while DLS was 2.3-7.5 nm, $83.32 \mathrm{~nm}, 89.8 \mathrm{~nm}$, and 100-1000 nm, respectively. In CS-NPs, the interaction between ammonium group of CS and polyphosphoric of TPP was determined by FTIR with peaks $\left(\mathrm{cm}^{-1}\right)$ of 3428, 1580 [57], 3288, 1647 [58], 1636, 3410 [51], 1648.84, 1527.35 [59], 1563 [60], and 3421.2 [53]. The crystal phase of CS-NPs is amorphous and has been identified by X-ray diffraction [58,59]. UV-Vis is not common in determining properties of CS-NPs synthesized according to the ionic gelation method. As reported by the authors of [59] and [54], CS-NPs absorb at wavelengths of 295 and $320 \mathrm{~nm}$, respectively. Interestingly, the authors of [63] synthesized CS-NPs by CS and anionic protein of Penicillium oxalicum by mass and volume ratio by 7.5:0.108 and 5:2 under stirring for $30 \mathrm{~min}$. Hydrodynamic size, dry state size, PDI, and zeta potential were $89.8 \mathrm{~nm}, 10-30 \mathrm{~nm}$ (spherical), 0.225 , and $-37 \mathrm{mV}$, respectively, and the peaks of $1602.8,1564.18$, and $1403.5 \mathrm{~cm}^{-1}$ characterized the binding of proteins and CS. These NPs absorb at wavelength of $285 \mathrm{~nm}$ and are amorphous.

To improve application efficiency, CS-NPs can load drugs and bioactive molecules (active ingredients), depending on the purpose. These NPs were synthesized by adding drugs and bioactive molecules solution to CS and TPP during the gelation process (incorporation) or after that (incubation) $[18,42,49,50]$. This is shown in Table 2 . The active ingredient 
can be metal ion ( $\mathrm{Cu}, \mathrm{Ag}, \mathrm{Zn}, \mathrm{Mn}$, and Fe) [32,51], drug (gentamicin-salicylic acid (SA) complex and Ag-Furosemide complex) [55,64], protein (Harpin from Pseudomonas syringae pv. syringae), agrochemical (Hexaconazole) [58], hormone (SA) [65], or other bio-molecules (saponin, thiamine, or Achillea millefolium extract) $[51,66,67]$. The mass ratio between the active ingredient and the CS or TPP can be smaller or larger, which affects the characters of the NPs. Metal ions (Ag, Cu, Zn, Mn, and Fe) are added to CS: TPP (1:10 or 15:2) at a rate such that the final ion concentration reaches $0.012 \%$ [32,51]. Ag-Furosemide complex was added to CS:TPP, with ratios of 0.005:10:2 and 0.01:10:2. The DLS of the two NPs was $210.5 \mathrm{~nm}$, PDI 0.232, and $41.5 \mathrm{mV}$; and $197.1 \mathrm{~nm}$, PDI 0.234 , and $36.7 \mathrm{mV}$, respectively [55]. When changing the mass ratio TPP:CS by 1:3, 1:4, 1:5, 1:6, and 1:7 (keeping the gentamicinsalicylic complex ratio), the DLS of NPs changed with decreasing size and increasing zeta potential $(343.3 \mathrm{~nm}$, PDI 0.41, $34.26 \mathrm{mV} ; 217.7 \mathrm{~nm}$, PDI 0.275, $35.77 \mathrm{mV} ; 180.0 \mathrm{~nm}$, PDI $0.235,37.12 \mathrm{mV}$; $172.2 \mathrm{~nm}$, PDI $0.308,39.44 \mathrm{mV}$; and $150.8 \mathrm{~nm}, 0.237 \mathrm{PDI}, 42.43 \mathrm{mV}$, respectively) [64]. On the other hand, the results of various mass ratios between TPP and CS:Hexaconazole by 1:5:10, 2:5:10, 4:5:10, and 8:5:10 showed that the sizes of the NPs decreased with respect to their ratios $(220.2,164.2,68.1$, and 6.5-18.1 nm, respectively) [58]. The Harpin protein (P. syringae pv. syringae) was also loaded into CS-NPs, with size of $133.7 \mathrm{~nm}$ and zeta potential at $48.6 \mathrm{mV}$, with an initial mass ratio of 1: 100: 20 [60]. When adding SA to CS: TPP at the ratio 1: $4: 2$, the DLS was $368.7 \mathrm{~nm}$, PDI 0.1, and $+34.1 \mathrm{mV}$ [65]. The authors of [51] and [66] added Saponin and Thiamine to CS:TPP at the ratio 1:2:20 and 25:24:4, and DLS of the two NPs was $373.9 \mathrm{~nm}$ (2 peaks), PDI 1.0, $+31 \mathrm{mV}$ and $596 \mathrm{~nm}$, $37.7 \mathrm{mV}$, respectively. In a study by [67], CS-NP was loaded with $A$. millefolium extract by mix the extract (semi-solid form) with $0.1 \%$ CS solution to obtain $20 \%$ before adding $1 \%$ TPP solution. This led to the formation of NPs with a size of $118 \mathrm{~nm}$ but containing 3 peaks $(10,122$, and $712 \mathrm{~nm})$. CS-NPs-loaded mental ions have a compact polyhedron shape, while CS-NPs-loaded saponin, SA, and gentamicin-SA complex were spherical when observed under SEM and TEM [51,64,65]. The size of CS-NPs-loaded active ingredient when recorded under TEM is sometimes larger than the size specified by DSL. CS-NPs-loaded hexaconazole with initial CS:TPP:hexaconazole ratios of 5:1:10 and 5:2:10 had dried state sizes at $271.4 \mathrm{~nm}$ and $168.5 \mathrm{~nm}$, while DLS is 220.2 and $164.2 \mathrm{~nm}$, respectively. However, at the ratio of 5:4:10 and 5: 8:10, the TEM sizes were 32.3 and $8.1 \mathrm{~nm}$, compared with DLS with 68.1 and $6.5-18.1 \mathrm{~nm}$ (2 peaks), respectively [58]. In contrast, CS-NPs-loaded thiamine was $596 \mathrm{~nm}$ by DLS but 10-60 nm by TEM [66]. Not only using TPP as anions, the authors of [68] also synthesized a novel conductive bio-composite membrane by combining CS and phosphotungstate anions on an aluminum substrate using the the ionic gelation method. Interaction in these NPs can also be determined by FTIR. CS-NPs-loaded $\mathrm{Cu}$ is characterized by peaks of 1631 and $1536 \mathrm{~cm}^{-1}$ for amide $\left(-\mathrm{CONH}_{2}\right)$ and primary amide $\left(-\mathrm{NH}_{2}\right)$, respectively [51]. Peaks of 1345 and $1095 \mathrm{~cm}^{-1}$ feature Harpin protein assigned to C-N and C-O stretch [60]. Peaks of 3218 and $3430 \mathrm{~cm}^{-1}$ characterize a hydrogen bonding between three chemicals in CS-NPs-loaded hexaconazole [58]. CS-NPs-loaded saponin was characterized by peak $3430 \mathrm{~cm}^{-1}$ for the hydrogen bonding between saponin and CS, and $1536 \mathrm{~cm}^{-1}$ for amide linkage between saponin and CS-NPs [51]. The peak at $1317 \mathrm{~cm}^{-1}$ featured an interaction between -COOH of SA and primary amide $\left(-\mathrm{NH}_{2}\right)$ of CS in a CS-NPs-loaded SA [65]. In CS-NPs-loaded gentamicin-SA complex, a peak at $3423 \mathrm{~cm}^{-1}$ characterizes the hydrogen bonding between -OH group bending of gentamicin and CS, two peaks of 1542 and $1637 \mathrm{~cm}^{-1}$ for interaction between $\mathrm{NH}_{3}{ }^{+}$of CS and TPP, and $1300 \mathrm{~cm}^{-1}$ for CN bending between $\mathrm{COOH}$ of SA and primary amide of CS [64]. Additionally, the peak of $1657 \mathrm{~cm}^{-1}$ characterizes the binding of thiamine and CS in these NPs [66]. The crystal phase of the NPs can also be identified by the X-ray diffraction technique. CS-NPs-loaded Ag-Furosemide complex was amorphous, while the crystalline peak of hexaconazole was clearly embedded in the amorphous phase of CS [58]. In addition, peak $2 \theta$ of $10^{\circ}-20^{\circ}$ and $18^{\circ}-30^{\circ}$ was recognized for SA and CS, respectively [65]. UV-Vis is seldom used, and only CS-NPs-loaded with $267 \mathrm{~nm}$ absorption thiamine was reported by [66]. In general, the main steps for synthesizing and building the CS-NPs-loaded active ingredients using the 
ionic gelation method are shown in Figure 3. Parameters can be optimized to suit each laboratory's conditions.

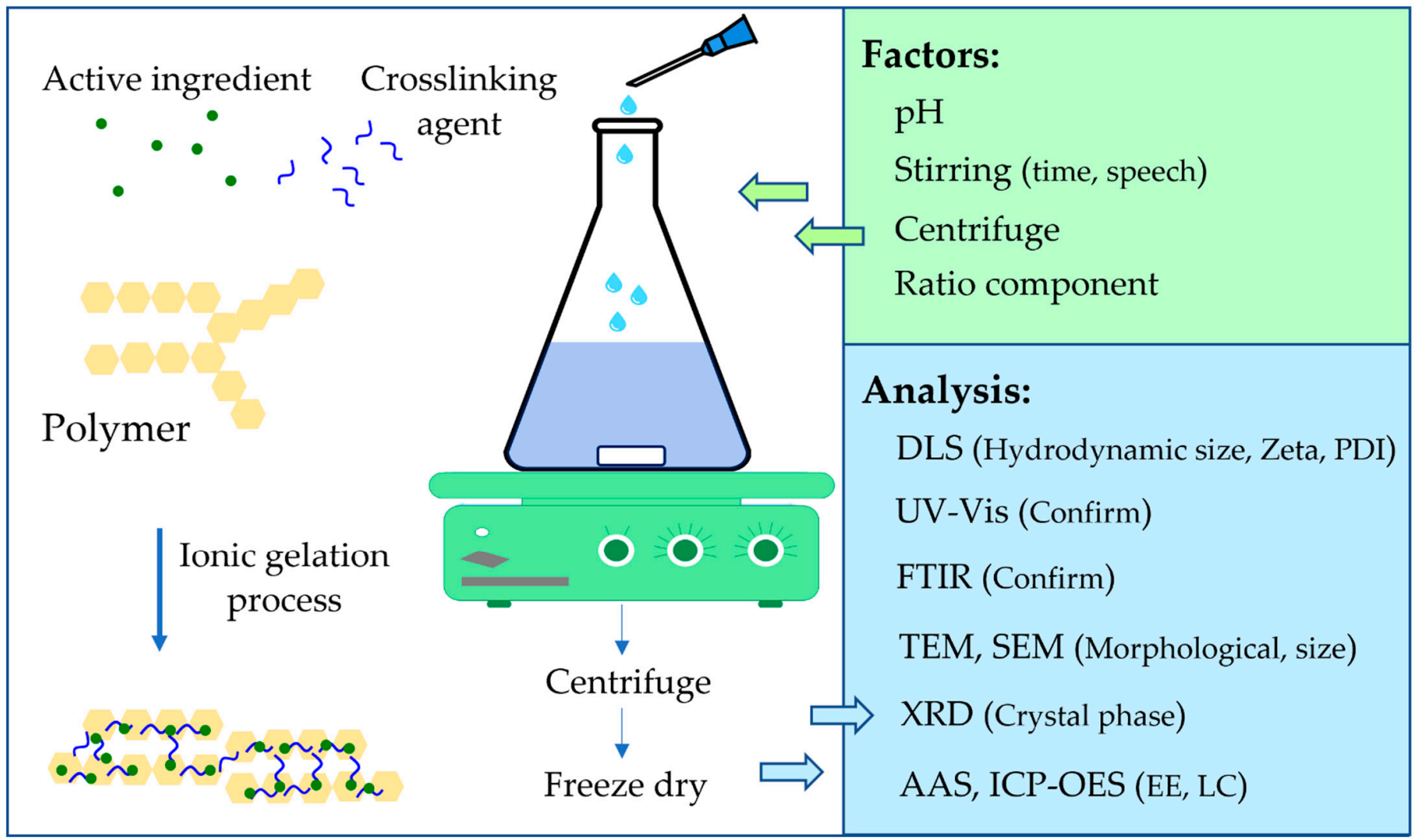

Figure 3. The schematic representation of nanoparticles synthesized and characterized by ionic gelation method.

The ionic gelation method requires simple, easy-to-find, and expensive materials and equipment, so it can be done easily, mildly, and quickly in normal laboratories. In addition, the mechanism based on electrostatic interaction instead of chemical reaction leads to no need to use organic solvents, thus avoiding potential toxicity of chemicals or reagents. However, the disadvantage of this method is that it is not easy to produce uniformly sized NPs, and research on other polymers (not CS) is limited [18,37,40,49]. 
Table 1. Character of CS-NPs synthesized by ionic gelation method.

\begin{tabular}{|c|c|c|c|c|c|c|c|c|}
\hline Mass Ratio & Volume Ratio & Condition Synthesis & DLS & SEM, TEM & FTIR $\left(\mathrm{cm}^{-1}\right)$ & $\mathrm{UV}(\mathrm{nm})$ & XRD & Reference \\
\hline $2: 5$ & $1: 10$ & $\begin{array}{c}\text { CS 0.2\%: TPP } 0.05 \%(1: 10), 25^{\circ} \mathrm{C}, \\
\mathrm{pH} 4\end{array}$ & $50 \mathrm{~nm}$ & Spherical & $\begin{array}{l}\text { 3428, 1580: hydrogen } \\
\text { bonding between } \\
\text { polyphosphoric of TPP } \\
\text { and ammonium group } \\
\text { of CS }\end{array}$ & - & - & [57] \\
\hline $3: 1$ & $3: 1$ & $\begin{array}{c}3 \mathrm{~mL} \text { of } 0.5 \% \mathrm{CS} \text { varying between }(\mathrm{A}) \\
\text { low molecular weight } \\
(\mathrm{Mw}=161 \mathrm{kDa}),(\mathrm{B}) \text { medium } \\
\text { molecular weight }(\mathrm{Mw}=300 \mathrm{kDa}), \\
(\mathrm{C}) \text { high molecular weight } \\
(\mathrm{Mw}=810 \mathrm{kDa}), \text { and } 1 \mathrm{~mL} \text { of } 0.5 \% \\
\text { TPP, centrifuge } 25,000 \mathrm{rpm} \text { for } 30 \mathrm{~min} \\
3 \mathrm{~mL} \text { of } 0.5 \% \mathrm{CS}(\mathrm{pH} 4.7-5) \text { varying } \\
\text { between }(\mathrm{D}) \text { low molecular weight } \\
(\mathrm{Mw}=161 \mathrm{kDa}),(\mathrm{E}) \text { medium } \\
\text { molecular weight }(\mathrm{Mw}=300 \mathrm{kDa}),(\mathrm{F}) \\
\text { high molecular weight } \\
(\mathrm{Mw}=810 \mathrm{kDa}), \text { and } 1 \mathrm{~mL} \text { of } 0.5 \% \\
\text { TPP, adjust pH to } 4.5-5 \text {, and discard } \\
\text { supernatant to collect CS-NPs }\end{array}$ & $\begin{array}{c}\text { (A) } 180.9 \mathrm{~nm}, \text { PDI } \\
0.31,45.6 \mathrm{mV} \\
\text { (B) } 309.9 \mathrm{~nm}, \text { PDI } \\
0.46,33.2 \mathrm{mV} \\
\text { (C) } 339.4 \mathrm{~nm}, \text { PDI } \\
0.52,21.7 \mathrm{mV} \\
\\
\\
\text { (D) } 225.7 \mathrm{~nm}, \text { PDI } \\
0.44,33.4 \mathrm{mV} \\
\text { (E) } 301.5 \mathrm{~nm}, \text { PDI } 0.2, \\
20.2 \mathrm{mV} \\
\text { (F) } 595.7 \mathrm{~nm}, \text { PDI } \\
0.92,16 \mathrm{mV}\end{array}$ & - & - & - & - & [52] \\
\hline $1: 10$ & $1: 1$ & $\begin{array}{c}0.1 \% \text { CS and } 1 \% \text { TPP with ratio } 1: 1 \\
\text { 10,000 rpm for } 10 \text { min and } \\
\text { ultrasonication }\end{array}$ & $\begin{array}{l}192.5 \mathrm{~nm} \\
\text { PDI } 0.6 \\
+45.33 \mathrm{mv}\end{array}$ & Spherical & $\begin{array}{c}\text { 1636, 3410: } \\
\text { hydrogen bonding }\end{array}$ & - & - & [51] \\
\hline $6: 1$ & $3: 1$ & $\begin{array}{l}0.5 \% \mathrm{CS} \text { (pH 5) and } 0.25 \% \text { TPP with } \\
\text { ratio 3:1, 10,000 rpm for } 10 \mathrm{~min}\end{array}$ & $\begin{array}{l}83.32 \mathrm{~nm} \\
\text { PDI } 0.31 \\
-28 \mathrm{mV}\end{array}$ & $\begin{array}{l}\text { Spherical } \\
20-50 \mathrm{~nm}\end{array}$ & $\begin{array}{c}\text { 1648.84, 1527.35: } \\
\text { interaction between } \\
\text { ammonium group of CS } \\
\text { and polyphosphoric } \\
\text { group of TPP }\end{array}$ & $295 \mathrm{~nm}$ & Amorphous & [59] \\
\hline $5: 1$ & $5: 1$ & $\begin{array}{c}5 \mathrm{~mL} \text { of } 0.1 \% \mathrm{CS}(\mathrm{pH} 5.5) \text { and } 1 \mathrm{~mL} \text { of } \\
0.1 \% \mathrm{TPP}, 20,000 \mathrm{rpm} \text { for } 30 \mathrm{~min}\end{array}$ & $\begin{array}{c}86.8 \mathrm{~nm} \\
+32.4 \mathrm{mV}\end{array}$ & Spherical & $\begin{array}{l}\text { 1563: interaction of } \\
\text { amide and phosphate }\end{array}$ & - & - & [60] \\
\hline $69.4: 1$ & $5: 2$ & $\begin{array}{l}15 \mathrm{~mL} \text { of } 0.5 \% \mathrm{CS}(\mathrm{pH} 4.8 \text { ) and } 6 \mathrm{~mL} \\
\text { of } 0.018 \% \text { anionic protein of } \\
\text { P. oxalicum, stirring } 30 \mathrm{~min} \text {, and } \\
\text { centrifuge } 10,000 \times \mathrm{g} \text { for } 10 \mathrm{~min}\end{array}$ & $\begin{array}{l}89.8 \mathrm{~nm} \\
\text { PDI } 0.225 \\
-37 \mathrm{mV}\end{array}$ & $\begin{array}{l}\text { Spherical } \\
10-30 \mathrm{~nm}\end{array}$ & $\begin{array}{c}\text { 1602.8, 1564.18, 1403.5: } \\
\text { binding of Protein } \\
\text { and CS }\end{array}$ & $285 \mathrm{~nm}$ & Amorphous & [63] \\
\hline
\end{tabular}


Table 1. Cont.

\begin{tabular}{|c|c|c|c|c|c|c|c|c|}
\hline Mass Ratio & Volume Ratio & Condition Synthesis & DLS & SEM, TEM & FTIR $\left(\mathrm{cm}^{-1}\right)$ & UV (nm) & XRD & Reference \\
\hline $\begin{array}{c}10: 1 \\
8: 1\end{array}$ & $\begin{array}{l}5: 1 \\
4: 1\end{array}$ & $\begin{array}{c}\text { 0.2\% Hydrolyzed CS (by chitinase } \\
\text { from Burkholderia cepacia E76) and } \\
0.1 \% \text { TPP with varying ratio (A) } 5: 1 \\
\text { and stirring } 60 \mathrm{~min}, \text { (B) } 5: 1 \text { and } \\
\text { stirring } 30 \mathrm{~min} \text {, (C) } 4: 1 \text { and stirring } \\
60 \mathrm{~min} \text {, and (D) } 4: 1 \text { and stirring } \\
30 \mathrm{~min}\end{array}$ & $\begin{array}{c}\text { (A) } 126.2 \mathrm{~nm}, \text { PDI } \\
0.44,27.8 \mathrm{mV} \\
\text { (B) } 167.1 \mathrm{~nm}, \text { PDI } \\
0.47,25.4 \mathrm{mV} \\
\text { (C) } 493.3 \mathrm{~nm}, \text { PDI } \\
0.69,22.9 \mathrm{mV} \\
\text { (D) } 573.1, \text { PDI } 0.54 \\
20.8 \mathrm{mV}\end{array}$ & Spherical-like & - & - & - & [56] \\
\hline $2: 5$ & $1: 1$ & $\begin{array}{c}0.1 \% \mathrm{CS}, 0.25 \% \mathrm{TPP} \text { with ratio } 1: 1, \\
\text { centrifuge } 10,000 \mathrm{rpm} \text { for } 10 \mathrm{~min} \text {, and } \\
\text { ultrasonication } 28 \% \text { pulse for } 100 \mathrm{~s} \\
\text { at } 4{ }^{\circ} \mathrm{C}\end{array}$ & $100-1000 \mathrm{~nm}$ & $\begin{array}{c}100 \mathrm{~nm} \\
\text { Spherical-like } \\
\text { High porous surface }\end{array}$ & $\begin{array}{c}\text { 1576: } \mathrm{NH}_{2} \text { bond } \\
\text { (wagging) } \\
\text { 1412: } \mathrm{C}-\mathrm{H} \text { bending } \\
\text { vibration of alkyl group }\end{array}$ & $320 \mathrm{~nm}$ & - & [54] \\
\hline $\begin{array}{c}5: 1 \\
10: 1 \\
15: 1 \\
20: 1\end{array}$ & $2: 1$ & $\begin{array}{c}10 \mathrm{~mL} \text { of } 0.1 \% \mathrm{TPP}, 20 \mathrm{~mL} \text { of CS } \\
\text { varying between (A) } 0.25 \% \text {, (B) } 0.5 \% \text {, } \\
\text { (C) } 0.75 \% \text { and (D) } 1 \% \text {; stirring } 8 \mathrm{~h}, \\
\text { and sonication } 45 \mathrm{~min}\end{array}$ & $\begin{array}{l}\text { (A) } 238.17 \mathrm{~nm} \\
\text { (B) } 575.2 \mathrm{~nm} \\
\text { (C) } 706.01 \mathrm{~nm} \\
\text { (D) } 1315.37 \mathrm{~nm}\end{array}$ & - & $\begin{array}{l}\text { 3421.2: interaction } \\
\text { between phosphate } \\
\text { and } \mathrm{NH}_{2}\end{array}$ & - & - & [53] \\
\hline $15: 2$ & $25: 1$ & $\begin{array}{c}25 \mathrm{~mL} \text { of } 0.3 \% \mathrm{CS}, 1 \mathrm{~mL} \text { of } 1 \% \mathrm{TPP} \\
\text { stirring } 20 \mathrm{~min} \text {, sonication } 1.5 \mathrm{~kW} \text { for } \\
30 \mathrm{~min} \text {, and centrifuge } 12,000 \times \mathrm{g} \\
\text { for } 10 \mathrm{~min}\end{array}$ & $\begin{array}{l}53.99 \mathrm{~nm} \\
\text { PDI } 1.0 \\
51.37 \mathrm{mV}\end{array}$ & - & - & - & - & [32] \\
\hline
\end{tabular}

Note: CS: chitosan; DLS: dynamic light scattering; FTIR: Fourier-transform infrared spectroscopy; NPs: nanoparticles; PDI: polydispersity index; SEM: scanning electron microscope TEM: transmission electron microscopy; TPP: sodium tripolyphosphate; UV: ultraviolet visible; XRD: X-ray diffraction. 
Table 2. Character of CS-NPs-loaded active ingredients synthesized by ionic gelation method.

\begin{tabular}{|c|c|c|c|c|c|c|c|c|c|}
\hline NPs & Mass Ratio & Volume Ratio & Condition Synthesis & DLS & SEM, TEM & FTIR $\left(\mathrm{cm}^{-1}\right)$ & UV (nm) & XRD & Reference \\
\hline $\begin{array}{l}\text { CS-NP-loaded } \\
\text { copper }(\mathrm{Cu})\end{array}$ & $1: 10$ & $1: 1$ & $\begin{array}{l}0.1 \% \text { CS and } 1 \% \text { TPP } \\
\text { with ratio } 1: 1 \text {, added } \\
0.01 \% \mathrm{CuSO}_{4} \text { to final } \\
\text { concentration of } \mathrm{Cu}^{2+} \\
0.012 \% \text { in mixture, } \\
10,000 \mathrm{rpm} \text { for } 10 \mathrm{~min}, \\
\text { and ultrasonication }\end{array}$ & $\begin{array}{l}196.4 \mathrm{~nm} \\
\text { PDI } 0.5 \\
+88 \mathrm{mv}\end{array}$ & $\begin{array}{l}\text { Compact } \\
\text { polyhedron }\end{array}$ & $\begin{array}{c}\text { 1631: }-\mathrm{CONH}_{2} \\
\text { 1536: }-\mathrm{NH}_{2}\end{array}$ & - & - & [51] \\
\hline $\begin{array}{l}\text { CS-NP-loaded } \\
\text { mental ion }(\mathrm{Ag}, \\
\mathrm{Cu}, \mathrm{Zn}, \mathrm{Mn}, \\
\text { and Fe) }\end{array}$ & $15: 2$ & $25: 1$ & $\begin{array}{c}25 \mathrm{~mL} \text { of } 0.3 \% \mathrm{CS}, 1 \mathrm{~mL} \\
\text { of } 1 \% \mathrm{TPP} \text {, and salt } \\
\text { solution at } 0.3 \% \text { added } \\
\text { to mixture to ion final } \\
\text { concentration } 0.012 \% ; \\
\text { stirring } 20 \mathrm{~min}, \\
\text { sonication } 1.5 \mathrm{~kW} \text { for } \\
30 \mathrm{~min} \text {, and centrifuge } \\
12,000 \times g \text { for } 10 \mathrm{~min}\end{array}$ & $\begin{array}{c}\text { (Ag) } 90.29 \mathrm{~nm}, \\
92.05 \mathrm{mV} \\
(\mathrm{Cu}) 121.9 \mathrm{~nm}, \\
88.69 \mathrm{mV} \\
(\mathrm{Zn}) 210.9 \mathrm{~nm}, \\
86.65 \mathrm{mV} \\
(\mathrm{Mn}) 102.3 \mathrm{~nm}, \\
75.74 \mathrm{mV} \\
\text { (Fe) } 95.81 \mathrm{~nm}, \\
71.42 \mathrm{mV} \\
\end{array}$ & - & - & - & - & [32] \\
\hline $\begin{array}{l}\text { CS-NP-loaded } \\
\text { Silver- } \\
\text { Furosemide } \\
\text { complex }\end{array}$ & 10:2:0.01 & $1: 2$ & $\begin{array}{c}10 \mathrm{~mL} \text { of } 1 \% \mathrm{CS}, 20 \mathrm{~mL} \\
\text { of } 0.1 \% \mathrm{TPP}, \mathrm{pH} 5.5, \\
\text { stirring } 1000 \mathrm{rpm} \text { for } 5 \\
\text { min, and sonication } 30 \% \\
\text { amplitude for } 10 \mathrm{~min} . \\
\text { (A) } 5,(\mathrm{~B}) 10 \mathrm{mg} \\
\text { Silver-Furosemide } \\
\text { complex was mixed with } \\
\text { TPP solution }\end{array}$ & $\begin{array}{c}\text { (A) } 210.5 \mathrm{~nm}, \mathrm{PDI} \\
0.232, \text { and } 41.5 \mathrm{mV} \\
\text { (B) } 197.1 \mathrm{~nm}, \mathrm{PDI} \\
0.234 \text {, and } 36.7 \mathrm{mV}\end{array}$ & - & - & - & Amorphous & [55] \\
\hline $\begin{array}{l}\text { CS-NP-loaded } \\
\text { Harpin } \\
\text { (P. syringae pv. } \\
\text { syringae) }\end{array}$ & 100:20:1 & $10: 2: 1$ & $\begin{array}{c}5 \mathrm{~mL} \text { of } 0.1 \% \mathrm{CS}(\mathrm{pH} 5.5) \\
\text { and } 1 \mathrm{~mL} \text { of } 0.1 \% \mathrm{TPP} \\
0.5 \mathrm{~mL} \text { of } 0.01 \% \text { Harpin, } \\
\text { and } 20,000 \mathrm{rpm} \text { for } \\
30 \mathrm{~min}\end{array}$ & $\begin{array}{l}133.7 \mathrm{~nm} \\
+48.6 \mathrm{mV}\end{array}$ & - & $\begin{array}{c}\text { 1345, 1095: Harpin } \\
\text { assigned to C-N } \\
\text { stretch and C-O } \\
\text { stretch in } \\
\text { CS-NP-loaded } \\
\text { Harpin }\end{array}$ & - & - & [60] \\
\hline $\begin{array}{l}\text { CS-NP-loaded } \\
\text { Hexaconazole }\end{array}$ & $\begin{array}{l}5: 1: 10 \\
5: 2: 10 \\
5: 4: 10 \\
5: 8: 10\end{array}$ & $5: 2: 5$ & $\begin{array}{c}100 \mathrm{~mL} \text { of } 0.5 \% \text { CS and } \\
100 \mathrm{~mL} \text { of } 1 \% \\
\text { hexaconazole, } 2 \% \\
\text { TWEEN } 80,40 \mathrm{~mL} \text { of } \\
\text { TPP varying between (A) } \\
0.25 \% \text {, (B) } 0.5 \%, \text { (C) } 1 \% \text {, } \\
\text { and (D) } 2 \%\end{array}$ & $\begin{array}{l}\text { (A) } 220.2 \mathrm{~nm} \\
\text { (B) } 164.2 \mathrm{~nm} \\
\text { (C) } 68.1 \mathrm{~nm} \\
\text { (D) } 2 \text { peaks } \\
\text { (6.5 and } 18.1 \mathrm{~nm} \text { ) }\end{array}$ & $\begin{array}{l}\text { (A) } 271.4 \mathrm{~nm} \\
\text { (B) } 168.5 \mathrm{~nm} \\
\text { (C) } 32.3 \mathrm{~nm} \\
\text { (D) } 8.1 \mathrm{~nm}\end{array}$ & $\begin{array}{l}\text { 3218: hydrogen } \\
\text { bonding of } \\
3 \text { chemicals }\end{array}$ & - & $\begin{array}{l}\text { Crystalline peak } \\
\text { of hexaconazole } \\
\text { clear embedded in } \\
\text { amorphous phase } \\
\text { of CS }\end{array}$ & [58] \\
\hline
\end{tabular}


Table 2. Cont.

\begin{tabular}{|c|c|c|c|c|c|c|c|c|c|}
\hline NPs & Mass Ratio & Volume Ratio & Condition Synthesis & DLS & SEM, TEM & FTIR $\left(\mathrm{cm}^{-1}\right)$ & $\mathrm{UV}(\mathrm{nm})$ & XRD & Reference \\
\hline $\begin{array}{l}\text { CS-NP-loaded } \\
\text { saponin }\end{array}$ & $2: 20: 1$ & 10:10:1 & $\begin{array}{c}0.1 \% \text { CS, } 1 \% \text { TPP and } \\
0.5 \% \text { saponin with ratio } \\
\text { 10:10:1, } 10,000 \text { rpm for } \\
10 \text { min, and } \\
\text { ultrasonication }\end{array}$ & $\begin{array}{c}373.9 \mathrm{~nm}(2 \text { peaks }) \\
\text { PDI } 1.0 \\
+31 \mathrm{mV}\end{array}$ & Spherical & $\begin{array}{l}\text { 1560: amide } \\
\text { linkage between } \\
\text { saponin and } \\
\text { CS-NPs } \\
\text { 3430: hydrogen } \\
\text { bonding between } \\
\text { saponin and CS }\end{array}$ & - & - & [51] \\
\hline $\begin{array}{l}\text { CS-NP-loaded } \\
\text { SA }\end{array}$ & $4: 2: 1$ & $1: 1: 1$ & $\begin{array}{l}0.4 \% \text { CS, } 0.2 \% \text { TPP and } \\
0.1 \% \text { SA with ratio } 1: 1: 1\end{array}$ & $\begin{array}{l}368.7 \mathrm{~nm} \\
\text { PDI } 0.1 \\
+34.1 \mathrm{mV}\end{array}$ & $\begin{array}{l}\text { Spherical and } \\
\text { porous }\end{array}$ & $\begin{array}{c}\text { 1541, 1639: } \\
\text { acetoxy group } \\
\text { of SA } \\
\text { 1317: interaction } \\
\text { between COOH } \\
\text { group of SA with } \\
\text { primary amide } \\
\text { of CS }\end{array}$ & & $\begin{array}{c}\text { Peak at } 2 \theta \text { of } \\
10^{\circ}-20^{\circ} \text { denoted SA } \\
\text { Peak } 2 \theta \text { of } 18^{\circ}-30^{\circ} \\
\text { confides CS }\end{array}$ & [65] \\
\hline \multirow[b]{2}{*}{$\begin{array}{l}\text { CS-NP-loaded } \\
\text { gentamicin } \\
(\mathrm{GM}) \text { and SA }\end{array}$} & - & - & $\begin{array}{c}0.1 \% \text { SA and } 0.2 \% \text { GM } \\
\text { with ratio } 3: 2,0.2 \% \text { CS } \\
\text { (pH 5). A mass TPP } \\
\text { solution added into CS } \\
\text { with ratio varying } \\
\text { between (A) } 1: 3,(\mathrm{~B}) 1: 4, \\
\text { (C) } 1: 5,(\mathrm{D}) 1: 6,(\mathrm{E}) 1: 7 ; \\
\text { stirring } 1 \mathrm{~h} \text {, and } \\
\text { centrifuge } 16,000 \mathrm{rpm} \\
\text { for } 30 \mathrm{~min}\end{array}$ & $\begin{array}{c}\text { (A) } 343.3 \mathrm{~nm}, \text { PDI } \\
0.41,34.26 \mathrm{mV} \\
\text { (B) } 217.7 \mathrm{~nm}, \text { PDI } \\
0.275,35.77 \mathrm{mV} \\
\text { (C) } 180.0 \mathrm{~nm}, \text { PDI } \\
0.235,37.12 \mathrm{mV} \\
\text { (D) } 172.2 \mathrm{~nm}, \text { PDI } \\
0.308,39.44 \mathrm{mV} \\
\text { (E) } 150.8 \mathrm{~nm}, \text { PDI } \\
0.237,42.43 \mathrm{mV}\end{array}$ & - & - & - & - & \multirow[b]{2}{*}{ [64] } \\
\hline & - & - & $\begin{array}{c}\text { CS/TPP ratio 4:1, pH 5.0, } \\
\text { drug-to-polymer ratio } \\
\text { 1:4, and feed ratio of SA } \\
\text { to GM 1.5:1.0. }\end{array}$ & $\begin{array}{l}180 \mathrm{~nm} \\
\text { PDI } 0.235 \\
37.12 \mathrm{mV}\end{array}$ & $\begin{array}{l}\text { Spherical } \\
200 \mathrm{~nm}\end{array}$ & $\begin{array}{c}\text { 3423: hydrogen } \\
\text { bonding between } \\
\text {-OH group } \\
\text { bending of } \mathrm{GM} \\
\text { and } \mathrm{CS} \\
1542,1637: \\
\text { interaction } \\
\text { between } \mathrm{NH}_{3}{ }^{+} \text {of } \\
\mathrm{CS} \text { and TPP } \\
\text { 1300: } \mathrm{C}-\mathrm{N} \text { bending } \\
\text { (interaction } \\
\text { between }-\mathrm{COOH} \\
\text { of } \mathrm{SA} \text { and primary } \\
\text { amide of } \mathrm{CS} \text { ) }\end{array}$ & - & - & \\
\hline
\end{tabular}


Table 2. Cont.

\begin{tabular}{|c|c|c|c|c|c|c|c|c|c|}
\hline NPs & Mass Ratio & Volume Ratio & Condition Synthesis & DLS & SEM, TEM & FTIR $\left(\mathrm{cm}^{-1}\right)$ & UV (nm) & XRD & Reference \\
\hline $\begin{array}{l}\text { CS-NP-loaded } \\
\text { Thiamine }\end{array}$ & 24:4:25 & $24: 8: 25$ & $\begin{array}{c}375 \mathrm{mg} \text { Thiamine } / 75 \\
\mathrm{~mL}, 360 \mathrm{mg} \mathrm{CS} / 72 \mathrm{~mL} \\
\text { and } 60 \mathrm{mg} \text { TPP } / 24 \mathrm{~mL} \text {, } \\
\text { stirring overnight, and } \\
\text { centrifuge } 10,000 \times g \text { for } \\
30 \mathrm{~min}\end{array}$ & $\begin{array}{l}596 \mathrm{~nm} \\
37.7 \mathrm{mV}\end{array}$ & $10-60 \mathrm{~nm}$ & $\begin{array}{l}\text { 1657: binding of } \\
\text { Thiamine to CS }\end{array}$ & $267 \mathrm{~nm}$ & - & [66] \\
\hline $\begin{array}{l}\text { CS-NP-loaded } \\
\text { A. millefolium } \\
\text { extract }\end{array}$ & 1:5:200 & 2:1:- & $\begin{array}{c}\text { A. millefolium extract } \\
\text { (semi solid form) added } \\
\text { into } 10 \mathrm{~mL} \text { of } 0.1 \% \mathrm{CS} \text { to } \\
\text { obtain final } \\
\text { concentration at } 20 \% \text {, } \\
5 \mathrm{~mL} \text { of } 1 \% \mathrm{TPP} \text {, stirring } \\
2 \mathrm{~h} \text { and centrifuge } \\
10,000 \times \mathrm{g} \text { for } 10 \mathrm{~min}\end{array}$ & $\begin{array}{l}118 \mathrm{~nm} \text { with } 3 \text { peaks } \\
(10,122 \text { and } 712 \mathrm{~nm})\end{array}$ & $\begin{array}{l}\text { Spherical } \\
4.15-100 \mathrm{~nm}\end{array}$ & $\begin{array}{l}\text { 3281.73, 2163.36 } \\
\text { and 1636.78: } \\
\text { interaction in NP }\end{array}$ & $417 \mathrm{~nm}$ & - & [67] \\
\hline $\begin{array}{l}\text { CS-NP-loaded } \\
\text { SA }\end{array}$ & $2: 1: 2$ & $1: 1: 1$ & $\begin{array}{l}0.4 \% \text { CS, } 0.2 \% \text { TPP, and } \\
0.2 \% \text { SA with ratio } 1: 1: 1\end{array}$ & $\begin{array}{l}89.86 \mathrm{~nm} \\
\text { PDI } 0.36 \\
22.27 \mathrm{mV}\end{array}$ & Spherical & $\begin{array}{l}\text { 3421: } \mathrm{NH}_{2} \text { stretch } \\
\text { 1640: } \mathrm{CO}^{-\mathrm{NH}_{2}} \\
\text { 1540: } \mathrm{NH}_{2} \text { bend } \\
\text { 1314: } \mathrm{COOH} \text { and } \\
\mathrm{NH}_{2} \\
\text { 895: Anhydro } \\
\text { glycoside }\end{array}$ & - & - & [69] \\
\hline $\begin{array}{l}\text { CS-NP-loaded } \\
\text { silver (Ag) }\end{array}$ & 5:5:0.51 & 1:1:1 & $\begin{array}{c}0.5 \% \mathrm{CS}, 0.5 \% \text { TPP, and } \\
3 \mathrm{mM} \text { silver nitrate with } \\
\text { ratio 1:1:1 }\end{array}$ & $\begin{array}{c}249 \mathrm{~nm} \\
\text { PDI } 0.53 \\
13.53 \mathrm{mV}\end{array}$ & Spherical & $\begin{array}{l}\text { 3423: } \mathrm{NH}_{2} \text { stretch } \\
\text { 1643: } \mathrm{CO}_{-} \mathrm{NH}_{2} \\
\text { 1542: } \mathrm{NH}_{2} \text { bend } \\
\text { 894: Anhydro } \\
\text { gly-coside }\end{array}$ & - & - & [69] \\
\hline
\end{tabular}

Note: CS: chitosan; DLS: dynamic light scattering; FTIR: Fourier-transform infrared spectroscopy; NPs: nanoparticles; PDI: polydispersity index; SEM: scanning electron microscope;

TEM: transmission electron microscopy; TPP: sodium tripolyphosphate; UV: ultraviolet visible; XRD: X-ray diffraction. 


\section{Application of CS-NPs-Based Ionic Gelation Method in Plant Disease Management}

With its advantages, CS-NPs synthesized according to the ionic gelation method has been applied in many fields, including pharmaceuticals, new materials, and agriculture (nanopesticides, nanofertilizers, and nanoherbicides) $[18,42,49,55,64,68]$.

For the management of plant diseases, CS-NPs can be applied as protectants (nano pesticides) and carriers (fungicides, insecticides, herbicides, plant hormones, elicitors, and nucleic acids) $[18,23,70]$. In particular, using CS-NPs as a delivery system is of special interest because it can load and protect the ingredients surrounding the environment and release them to the target site uptake of the plants [18]. In addition, with the basic properties of NPs having a small size and high contact area, CS-NPs or CS-NPs-loaded active ingredients can be easily penetrated and permeated into the membrane of phytopathogens or enhanced plant tissues uptake, resulting in an increased control or defense response activity, respectively [49]. Therefore, these NPs can be used directly and indirectly to manage plant diseases.

Studies using CS-NPs synthesis by ionic gelation method in plant disease management are shown in Table 3 and Figure 4.

\subsection{Chitosan Nanoparticles (CS-NPs) \\ 3.1.1. Directly}

Under in vitro conditions, the authors of [52] determined that the minimum inhibitory concentration of CS-NPs prepared by centrifuge and $\mathrm{pH}$ change method at 0.05 and $0.09 \%$ could inhibit growth of $F$. graminearum at $31.97 \%$ and $29.67 \%$, respectively. Furthermore, the authors of [52] also showed that CS-NPs were originated from CS low molecular weight, which has a higher inhibitory effect than CS height molecular weight at the same concentration. CS-NPs may or may not inhibit pathogens. CS-NPs may inhibit mycelial growth of Pyricularia grisea (65\%) at 0.1\% [71], Colletotrichum gloeosporioides (85.7\%) [56], C. gloeosporioides (37.8\%), Phytophthora capsica (50.7\%), Sclerotinia sclerotiorum $(39.5 \%)$, Fusarium oxysporum (50.3\%), Gibberella fujikuori (56.3\%) at 0.5\% [54], P. grisea (92\%), Alternaria solani (87\%), F. oxysporum (72\%) with amount of $100 \mu \mathrm{g}$ [63], A. alternata (80.1-82.2\%), R. solani (32.2-34.4\%) at $0.06-0.1 \%$, M. phaseolina (84\%) at $0.1 \%$ [51], A. solani $(10,70 \%)$ at 0.03, 0.04\% [72], Alternaria tenuis (67.67\%), Aspergillus niger (62.75\%), Aspergillus terreus $(74.67 \%)$, Baeuvaria bassiana $(76.08 \%)$, F. graminearum $(60.37 \%)$, F. oxysporum $(66.60 \%)$, Sclerotium rolfsii (37.41\%) at $800 \mathrm{ppm}$, and the zearalenone produced by F. graminearum [73]. Furthermore, CS-NPs were $0.014 \%$ (in acetate buffer), the lysis zone diameter of Clavibacter michiganensis and Fusarium graminearum were 29.5 and $20.0 \mathrm{~mm}$, and CS was 22.5 and $18.0 \mathrm{~mm}$, respectively [72]. Moreover, $0.2 \mathrm{~mL}$ of CS-NP at $125 \mathrm{ppm}$ could inhibit mycelium of $F$. graminearum by $44.3 \%$, higher than fungicide (8-hydroxy quinoline) at $42.33 \%$ [73]. In addition, CS-NPs also inhibited spore germination of $C$. gloeosporioides $(61.2 \%)$ [56] and A. alternata $(84.4-87.1 \%)$ at $0.06-0.1 \%$ [51]. On the other hand, CS-NPs do not inhibit mycelial growth, spore germination, and sporulation of $P$. grisea, even at a concentration of $0.1 \%$ [59,71]. A study by [54] showed that $\mathrm{OD}_{600 \mathrm{~nm}}$ of Erwinia carotovora subsp. carotovora strains 113114, 113154, and YKB133061, and Xanthomonas campestris pv. vesicatoria strain 11,154 , were reduced by $41.3,55.5,48.5$, and $52.1 \%$ when treated with CS-NPs at $0.5 \%$; interestingly, they were also reduced by $64.7,76.3,78.0$, and $73.8 \%$ when CS-NPs were treated at $0.05 \%$, respectively. Furthermore, CS-NPs at $2 \mathrm{~mL} / \mathrm{L}$ inhibited anthracnose disease at 87.5 and $75 \%$ for chili and at 50 and $10 \%$ for papaya, by using the preventative and curative treatments under in vivo conditions, respectively [56].

\subsubsection{Indirectly}

Pre-treatment of CS-NPs at $0.1 \%$ reduced sheath blight and blast in rice caused by R. solani and P. grisea by $92.78 \%$ and $100 \%$ under detach leaves assay, respectively $[59,74]$.

In the greenhouse trial, CS-NPs are capable of protecting plants of rice, finger millet, and wheat from pathogens attacks $[52,71,74]$. The sheath blight disease was reduced by 
$75.01 \%$ compared with CS at $44.82 \%$, and the peroxidase, phenylalanine ammonia-lyase, and chitinases activity also increased by 19-, 1.5-, and 1.9-fold, respectively [74]. In the study of [71], the symptom and disease incidence of blast was delayed by 10 days and decreased 2.8-fold, respectively, influenced by peroxidase activity (which increased 1.6-fold) and reactive oxygen species activity. Spray of CS-NPs at $0.05 \%$ after infection of F. graminearum leads to reduce AUPDC at 28 days after inoculation (DAI) by 2.2-fold compared to the water control. The NPs caused structural damage in mycelium and cell pathogen but also increased superoxide and $\mathrm{H}_{2} \mathrm{O}_{2}$ content [52].

\subsection{Chitosan-Nanoparticles-Loaded Active Ingredients}

\subsubsection{Directly}

The effect of controlling or enhancing the immunity of plants is different, depending on the same CS-NPs and the type of active ingredient. The $\mathrm{EC}_{50}$ of four formulate CSNPs-loaded hexaconazole to control Ganoderma boninense is $8.0-18.4$ ppb, which is 21.4 and $1534.5 \mathrm{ppb}$ lower than hexaconazole and CS-NPs, respectively. Similarly, fiducial limit (lower-upper) was 6.0-10.9 to $13.0-32.8 \mathrm{ppb}$, while hexaconazole and CS-NPs were 16.7-27.3 and 494.0-13280.4 ppb [58].

CS-NPs-loaded $\mathrm{Cu}$ could inhibit mycelial growth of Curvularia lunata by 50.0 and $52.7 \%$ at 0.12 and $0.16 \%$ [75], A. solani and F. oxysporum by 84.2 and $60.1 \%$ at $0.1 \%$ [76], A. alternata and $R$. solani by $82.1-89.5 \%$ and $62.5-63.0 \%$ at $0.06-0.1 \%$, and $M$. phaseolina by $60.1 \%$ at $0.1 \%$ [51], respectively. These NPs also inhibited spore germination of $A$. solani and F. oxysporum by 73.3 and $79.9 \%$ at $0.1 \%$ [75] and A. alternata by $83.3-87.4 \%$ at $0.06-0.1 \%$ [51]. CS-NPs-loaded Zn inhibited mycelial growth and spore germination of C. lunata by $47.7-65.2 \%$ and $50.5-73.3 \%$ at $0.08-0.16 \%$ [77]. In addition, the mixture of CS-NPs (ionic gelation) and Cu-NPs (chemical reduction) inhibited the mycelial growth of F. oxysporum by $61.94-100 \%$ at $0.05-0.2 \%$ [57]. CS-NPs-loaded SA evaded mycelial growth by $62.2-100 \%$ and spore germination of Fusarium verticillioides by $48.3-60.5 \%$ at $0.08-0.16 \%$ [65]. CS-NPs-loaded saponin inhibited mycelial growth of $A$. alternata by $78.3-80.9 \%$ and $R$. solani by $27.7 \%$ at $0.06-0.1 \%$ and spore germination of $A$. alternata by $78.3-82.9 \%$ at $0.1 \%$ [51]. On the other hand, CS-NPs-loaded thiamine did not inhibit F. oxysporum, even at a concentration of $0.1 \%$ [66].

Under greenhouse conditions, at 3 DAI, A. solani and F. oxysporum, CS-NPs-loaded $\mathrm{Cu}(0.1$ and $0.12 \%)$ was foliar sprayed and applied to soil lead to reduced early blight $(84.2$ and $87.7 \%$ ) and fusarium wilt (49.9 and 61.1\%), respectively [75]. Furthermore, priming maize seeds into these NPs $(0.02-0.14 \%)$ for 4 and $8 \mathrm{~h}$ combined with foliar spraying after $F$. verticillioides infected reduced post-flowering stalk rot disease by $38.2-48.1 \%$ and 24.8-49.6\%, respectively [78]. Moreover, these treatments reduced disease severity by $23.5-33.9 \%$ and $2.55-15.8 \%$ for 4 and $8 \mathrm{~h}$ priming under field conditions.

\subsubsection{Indirectly}

Previously, Harpin protein (from Erwinia amylovora) was known for its ability to induce systemic acquired resistance in plants [79]. With the same amount $(20 \mu \mathrm{g}), \mathrm{CS}-\mathrm{NPs}-$ loaded Harpin protein (from P. syringae pv. syringae) enhanced cell death, necrotic lesions, and $\mathrm{H}_{2} \mathrm{O}_{2}$ accumulation faster and stronger than Harpin protein only [60]. Furthermore, treatment of these NPs reduced fungal biomass (5-fold) and lesion diameter (12-fold) and caused failing colonization of $R$. solani in tomato leaves compared with the control. Peroxidase and phenylalanine ammonia-lyase activity also steadily increased up to $72 \mathrm{~h}$. Interestingly, the transcriptome changes, including defense response, signal transduction, transport, transcription, photosynthesis, housekeeping, and aromatic biosynthesis, were enhanced more than 2 -fold at 24, 48, and $72 \mathrm{~h}$ after spraying.

Under greenhouse conditions, pre-treated CS-NPs-loaded Cu (0.04-0.16\%) reduced leaf spot disease (C. lunata) in maize by 43.86-48.48\%. Moreover, this treatment increased superoxide dismutases (1.8-2.2 folds), peroxidase (1.5-2.1 folds), phenylalanine ammonialyase (1.3-2.0 folds), and polyphenol oxidase (1.1-1.2 folds) [75]. Furthermore, CS-NPs- 
loaded $\mathrm{Zn}$ also induced superoxide dismutases, phenylalanine ammonia-lyase, polyphenol oxidase, and $\mathrm{H}_{2} \mathrm{O}_{2}$ activity by 1.2-2.0-, 2.0-3.0-, 17.24-49.37-, and 1.5-2.6-fold when compared with the control, respectively. The $\mathrm{H}_{2} \mathrm{O}_{2}$ and lignin localization also increased, leading to maize leaf spot (C. lunata) reduction by 32.3-50.77\% [77].

A hormone-elicitor is an SA that has been loaded into CS-NPs. Maize was pre-treated with these NPs at $0.01-0.16 \%$ and suppressed post-flowering stalk rot disease (37.33-49.5\%) caused by F. verticillioides. Furthermore, at 2 and 3 days after spraying NPs, superoxide dismutases (1.8- and 3.2-fold), peroxidase (7.0- and 4.6-fold), catalase (3.1- and 2.6-fold), phenylalanine ammonia-lyase (2.0- and 1.7-fold), polyphenol oxidase (1.7- and 2.0-fold), $\mathrm{O}_{2}{ }^{-}$(1.1- and 1.1-fold), $\mathrm{H}_{2} \mathrm{O}_{2}$ (17.5- and 37.0-fold), and lignin accumulation increased [65]. Pre-treated CS-NP-loaded SA at 400 ppm and CS-NP-loaded Ag at 200, 400, and 800 ppm by stalk-soaking and foliar spraying reduced cassava leaf spot disease by $68.9-73.6 \%$ at 56 DAP (first inoculate with fungal density $10^{4}$ conidia per $\mathrm{mL}$ ) and 37.0-37.7\% at 75 DAP (second inoculate with fungal density $10^{5}$ conidia per $\mathrm{mL}$ ) [69].

Although CS-NPs-loaded thiamine did not inhibit fungi in vitro condition, pre-treatment of these NPs $(0.1 \%)$ at 3 days before infection of F. oxysporum on chickpea reduced cell death in 2 DAI compared with control. Furthermore, polyphenol oxidase, peroxidase, $\beta-1,3-$ glucanase, chitinase, chitosanase, and protease were increased by 2.1-, 2.0-, 1.4-, 1.4-, 1.4-, and 1.1-fold in leaves and 2.0-, 1.3-, 1.1-, 1.3-, 1.3-, and 1.1-fold in roots, respectively [66].

On the other hand, in the study of [80], CS-NPs-loaded Cu at $0.12-0.06 \%$ was treated before and after an infection of Xanthomonas axonopodis pv. glycine could reduce bacterial pustule disease in soybean by $40.6-49.7 \%$, respectively. Interestingly, the low concentration is more effective. In addition, application of the mixture of CS-NPs (ionic gelation) and $\mathrm{Cu}-\mathrm{NPs}$ (chemical reduction) to date palm root zone increased plant immunomodulatory, including total phenols (1.1-1.5 folds), phenoloxidases (1.1-2.0 folds), and peroxidase (1.6-3.0 folds), which led to a reduction in disease by 16.2-59.3\% [57].

Under field conditions, CS-NPs-loaded $\mathrm{Cu}, \mathrm{Zn}$, and SA are effective in reducing disease by inducing plant defense system in maize and soybean $[65,75,77,80]$. Treatment CS-NPs-loaded $\mathrm{Cu}(0.06 \%)$ reduced bacterial pustule disease by $51.3 \%$. In addition, these NPs at $0.01-0.08 \%$ reduced maize leaf spot disease by $27.72-28.53 \%$ while at $0.12-0.16 \%$ they reduced it by 30.42-33.8\% [75]. On the other hand, CS-NPs-loaded Zn at 0.01-0.16\% reduced this disease by $25.42-39.67 \%$ [77]. CS-NPs-loaded SA at $0.01-0.16 \%$ reduced post-flowering stalk rot by $40.5-59.47 \%$ [65].

\subsection{Plant Growth Promotion}

A concern for any agrochemical is the safety of plants, environment, farmers, and consumers. In recent reviews, NP is a biosafety solution. However, nanotoxicology still remains to be noticed $[81,82]$. When applying NPs to plants, they will enter the tissues and cause positive and negative impacts depending on their size, shape, and concentration. NPs usually enhance shoot elongation, root elongation, seed germination at low concentration, and in contrast at high concentration [17]. The effective concentration varies between NPs and crops. In the study by the authors of [69], the CS-NP-loaded SA and silver were tested for phytotoxicity with the cassava by leaf disk assay method before being applied to cassava plants at net house condition. Results showed that these formulations did not cause damage in leaf disk up to $800 \mathrm{ppm}$. Then, researchers varied concentrations of 25-800 ppm for stalk-soaking and foliar spraying to enhance cassava growth and reduce leaf spot disease. This is an easy way to know what "safe" concentrations are for the plant. When applied to soil, NPs can cause negative impacts on soil microflora but will be less damaging than agrochemical applications [83]. On the other hand, the amount of agrochemical and fertilizer applied to agriculture is reduced if they are replaced by NPs, which leads to a reduction in their toxicity. Usually, the safety-by-design principle is applied to screen potential risks from materials and methods synthesis to NP formulation [84]. As mentioned above, ionic gelation method and CS - a natural polymer-are friendly, safe, and biodegradable solutions. 
In addition to its ability to directly inhibit pathogens or induce plant defense system against diseases, CS-NPs or CS-NPs-loaded active ingredients have the ability to stimulate plant growth. At this time, they act as fertilizers or nutrients, affecting plant physiological processes, including nutrient uptake, cell division, cell elongation, enzymatic activation, and the synthesis of protein that leads to increase yield [43]. Efficiency depends on both the CS-NPs and the active ingredient, even when it releases all the active ingredients because the main component of CS is nitrogen, which takes 9-10\% [46]. Furthermore, the rich positive charge of CS leads to increased affinity toward the plant cell membrane, which enhances reactivity in the plant system [49].

Several types of NPs presented in Table 3 have been shown to stimulate plant growth.

In the seeding stage, CS-NPs increased the seeding vigor index (57.1\%), the number of lateral root (133.3\%), and dry weight (200\%) of chickpeas [63]. Additionally, the chickpea seeds were soaked with CS-NPs-loaded thiamine at $0.1 \%$ overnight, leading to the seeding vigor index increasing by $64.2 \%$, with Indole-3-acetic acid content increased 10-fold [66]. Treatment with CS-NPs-loaded $\mathrm{Cu}$ at $0.08,0.1$, and $0.12 \%$ increased seedling vigor index $(33.9,33.7$, and $24.3 \%)$, fresh weight $(18.9,21.6$, and $16.2 \%)$, and dry weight $(20.0,26.7$, and $13.3 \%$ ) in tomato, respectively [75]. Additionally, CS-NPs-loaded Cu at 0.01-0.16\% increased seeding vigor index (15.6-48.6\%), fresh weight (7.1-11.4\%), and dry weight $(21.4-57.1 \%)$ in maize seedings, which were related to increasing $\alpha$-amylase and proteases at days 5 and 7 , respectively [85].

Under greenhouse conditions, the dry weight and yield of finger millet increased by $148.8 \%$ and $93.2 \%$ when treated with CS-NPs, respectively [71]. The plant height, stem height, and root length of maize increased by 30.3-60.3, 66.3-237.5, and 2.7-61.1\% when treated with CS-NPs-loaded Zn at $0.01-0.16 \%$, respectively [77]. In chickpea, the shoot length, number of leaves per plant, fresh weight, dry weight, and number of secondary roots per plant increased by 15.3, 14.4, 37.7, 20.0, and 52.8\% when sprayed with CS-NPsloaded thiamine at $0.1 \%$, respectively [66]. In present year, pre-treated CS-NP-loaded SA at 400 ppm and CS-NP-loaded Ag at 200, 400, 800 ppm by stalk-soaking for 5 min and foliar spraying at 28, 42 DAP could increase the number of leaves (45.1-82.4\%), the number of shoots (38.5-46.2\%), the largest leaf area (29.6-41.9\%), root length (11.6-29.9\%), and root weight (27.6-82.8\%) of cassava, at 75 DAP in net house condition [69]. In addition, CS-NPs-loaded $\mathrm{Cu}$ at $0.06 \%$ could increase plant height $(56.8 \%)$, root length $(40.3 \%)$, and pod number $(7.2 \%)$. NPs treatment at $0.02 \%$ could increase root weight $(46.8 \%)$, nodule number $(44.2 \%)$, nodule weight $(125.8 \%)$ under greenhouse conditions and also increase root length $(60.9 \%)$, root weight $(46.8 \%)$, and pod number $(29.7 \%)$, in soybean under field conditions [80]. Furthermore, NPs at $0.01-0.08 \%$ increased plant height (15.9-47.0\%), stem diameter (82.9-102.9\%), root length (9.5-15.8\%), root number (20.9-46.3\%), and chlorophyll content (67.3-182.6\%) under greenhouse conditions and increased grain yield (25.4-29.3\%), 100 grain weight (14.4-16.9\%) in maize under field conditions [75]. However, the treatment at $0.16 \%$ reduced root length $(9.8 \%)$ and chlorophyll content $(4.6-9.7 \%)$, although the difference was not significance. The CS-NPs-loaded SA at $0.01-0.16 \%$ increased leaf area $(160.6-224.7 \%)$, shoot length (38.5-76.9\%), root length $(66.9-111.5 \%)$, root number (59.6-91.8\%), stem diameter (22.8-53.9\%), and total chlorophyll (54.2-141.4\%) in maize under greenhouse conditions. Moreover, the treatment of these NPs at $0.08 \%$, days to $50 \%$ tasselling was early by 4 days under field conditions. Moreover, the plant height (25.5\%), ear height $(12.1 \%)$, cob length $(44.8 \%)$, test weight $(71.1 \%)$, and grain yield $(48.3 \%)$ also increased [65]. The authors of [86] synthesized CS-NPs-loaded NPK (ionic gelation) with slow-release N (66.7\%), P (3.1\%), and K (57.7\%) for $240 \mathrm{~h}$. The leaf number, leaf area, plant height, and stem diameter of coffee increased 22.8, 46.9, 12.7, and 28.3\% when these NPs were treated at $30 \mathrm{ppm}$, respectively. This synthesized CS-NPs-loaded NPK improved N (17.04\%), P (13.1\%), K (67.5\%), chlorophyll (30.68\%), carotenoid (21.4\%) content, and photosynthesis rate $(71.7 \%)$ in the coffee leaves. Another nano fertilizer, CS-NPs-loaded silicon at $0.04-0.12 \%$, increased the seeding vigor index in maize seeding by $167.5-285.2 \%$. Furthermore, foliar spraying induced antioxidant defense enzyme activity; equilibrated 
cellular redox; and balanced $\mathrm{O}_{2}{ }^{-}$and $\mathrm{H}_{2} \mathrm{O}_{2}$ in leaf, leading to homeostasis. In the field trial, the yield and test weight of maize was increased by 186.6 and $77.1 \%$ by treated CS-NPs-loaded silicon at 0.08 and $0.04 \%$, respectively [87].

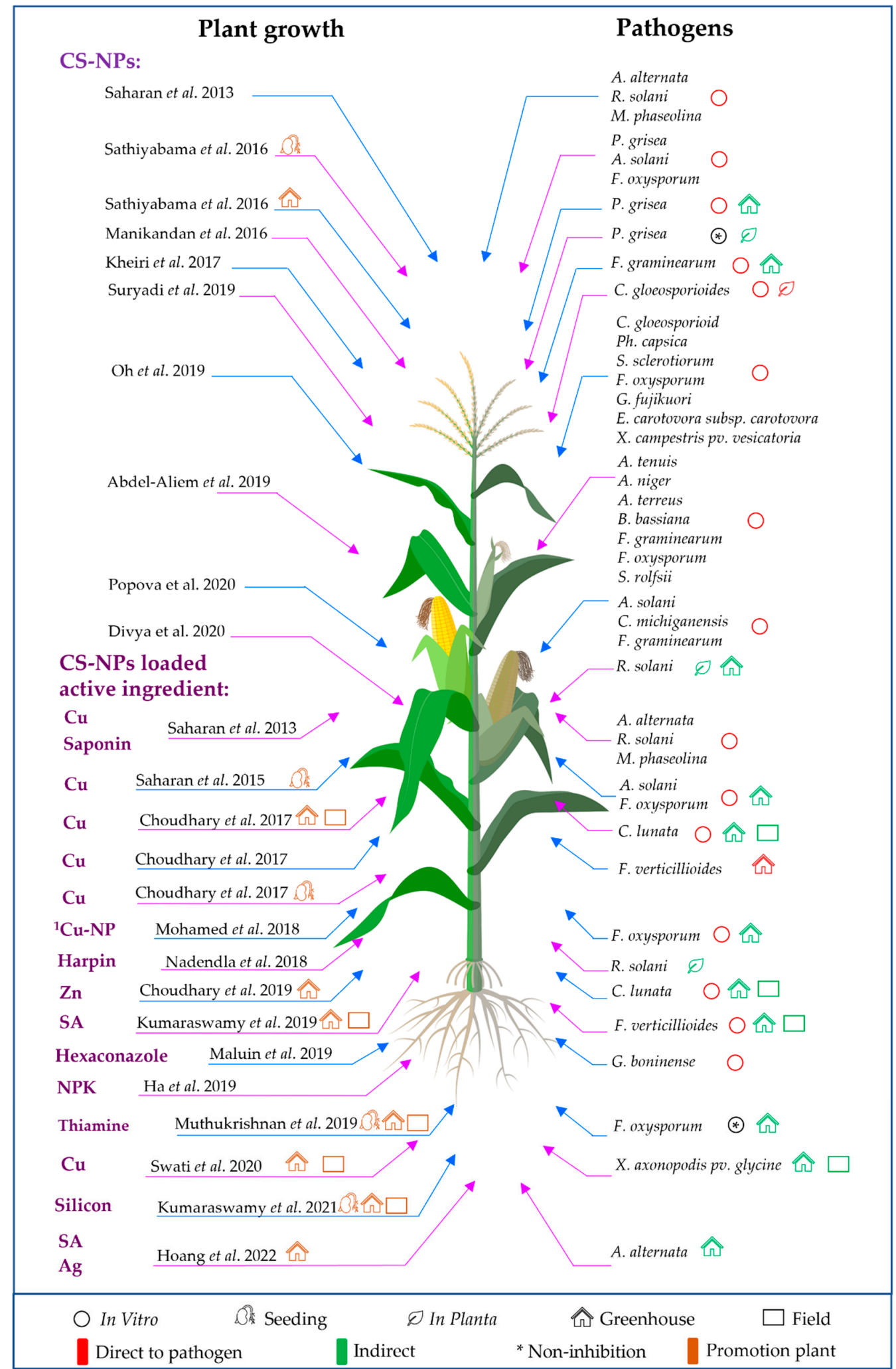

Figure 4. The application of CS-NPs and CS-NPs-loaded active ingredients synthesized by ionic gelation method in plant disease management and enhancing plant growth. Note: ${ }^{1}$ Mixture of CS-NP 
(ionic gelation method) and Cu-NP (chemical reduction method). Saharan et al. 2013 [51], Sathiyabama et al. 2016 [63], Sathiyabama et al. 2016 [71], Manikandan et al. 2016 [59], Kheiri et al. 2017 [52], Suryadi et al. 2019 [56], Oh et al. 2019 [54], Abdel-Aliem et al. 2019 [73], Popova et al. 2020 [72], Divya et al. 2020 [74], Saharan et al. 2015 [76], Choudhary et al. 2017 [75], Choudhary et al. 2017 [78], Choudhary et al. 2017 [85], Mohamed et al. 2018 [57], Nadendla et al. 2018 [60], Choudhary et al. 2019 [77], Kumaraswamy et al. 2019 [65], Maluin et al. 2019 [58], Ha et al. 2019 [86], Muthukrishnan et al. 2019 [66], Swati et al. 2020 [80], Kumaraswamy et al. 2021 [87], Hoang et al. 2022 [69].

Table 3. The CS-NPs synthesized by ionic gelation used in plant disease management.

\begin{tabular}{|c|c|c|c|c|}
\hline NPs & Plant & Pathogen & Summary Research & Reference \\
\hline $\begin{array}{l}\text { CS-NPs with DLS } \\
(83.32 \mathrm{~nm}, \text { PDI 0.31, } \\
-28 \mathrm{mV}), \text { HRTEM } \\
(20-50 \mathrm{~nm}) .\end{array}$ & Rice & $\begin{array}{l}\text { P. grisea } \\
\text { /Blast }\end{array}$ & $\begin{array}{l}\text { In vitro: Treat CS-NPs not cause inhibit } \\
\text { mycelial and spore germination even } 0.1 \% \text {. } \\
\text { Detach leaves assay: painting brush } \\
500 \mu \mathrm{L} \text { onto surface each leave. After } 24 \mathrm{~h} \text {, } \\
\text { inoculate with similar method. } \\
\text { Treating CS-NPs } 0.1 \% \text { could prevent blast } \\
\text { symptoms up to } 10 \mathrm{DAI} \\
\text { (suppression } 100 \% \text { ). }\end{array}$ & [59] \\
\hline $\begin{array}{c}\text { CS-NPs described } \\
\text { as [59] }\end{array}$ & Fingermillet & $\begin{array}{l}\text { P. grisea } \\
\text { /Blast }\end{array}$ & $\begin{array}{l}\text { In vitro: CS-NPs at } 0.1 \% \text { inhibited nearly } \\
65 \% \text { mycelial growth and did not } \\
\text { sporulate compared with the control. } \\
\text { Greenhouse: seed was soaked overnight, } \\
\text { foliar sprayed at } 20 \text { and } 30 \text { DAP, and } \\
\text { inoculated at } 30 \text { DAP. } \\
\text { Treatment with CS-NPs delay symptom } \\
\text { by } 10 \text { days and decreased disease } \\
\text { incidence } 2.8 \text {-fold. Moreover, treatment } \\
\text { with CS-NPs increased dry weight } \\
(148.8 \%) \text {, yield ( } 93.2 \%) \text {, peroxidase } \\
(1.6-\text { fold), and reactive oxygen } \\
\text { species activity. }\end{array}$ & [71] \\
\hline $\begin{array}{l}\text { CS-NPs with DLS } \\
(9.8 \mathrm{~nm}, \text { PDI 0.225, } \\
-37 \mathrm{mV}), \text { HRTEM } \\
(10-30 \mathrm{~nm}) .\end{array}$ & Chickpea & $\begin{array}{c}\text { P. grisea } \\
\text { A. solani } \\
\text { F. oxysporum }\end{array}$ & $\begin{array}{l}\text { In vitro: CS-NPs with amount } 100 \mu \mathrm{g} \\
\text { inhibited mycelial growth of } P \text {. grisea, } \\
\text { A. solani, F. oxysporum by } 92,87, \\
\text { and } 72 \% \text {, respectively. } \\
\text { Seeding: Treatment with NPs increased } \\
\text { seedling vigor index }(57.1 \%), \text { number } \\
\text { lateral root }(133.3 \%) \text {, and dry } \\
\text { weight }(200 \%) \text {. }\end{array}$ & [63] \\
\hline $\begin{array}{c}\text { CS-NPs } \\
\text { Centrifuge method } \\
\text { with DLS }(180.9 \mathrm{~nm} \text {, } \\
\text { PDI 0.31, and } 45.6 \mathrm{mV}) \text {. } \\
\text { pH change method } \\
\text { with DLS }(225.7 \mathrm{~nm} \text {, } \\
\text { PDI } 0.44 \text {, and } 33.4 \mathrm{mV}) \text {. }\end{array}$ & Wheat & $\begin{array}{l}\text { F. graminearum } \\
\text { /Head blight }\end{array}$ & $\begin{array}{l}\text { In vitro: CS-NPs synthesized by CS low } \\
\text { molecular weight significantly inhibited } \\
\text { pathogens, more than low molecular } \\
\text { weight and high molecular weight at the } \\
\text { same concentration. MIC of CS-NPs } \\
\text { prepared by centrifuge and pH change } \\
\text { method were } 0.05 \% \text { and } 0.09 \% \text { with } \\
31.97 \% \text { and } 29.67 \% \text {, respectively. } \\
\text { Greenhouse: Spraying after inoculate } \\
\text { The area under the disease progress curve } \\
\text { at } 28 \text { DAI of treatment CS-NPs } 0.05 \% \text { was } \\
\text { reduced } 2.2 \text {-fold compared with } \\
\text { the control. } \\
\text { The CS-NPs caused structural damage in } \\
\text { mycelium and cell pathogen and } \\
\text { increased superoxide and } \mathrm{H}_{2} \mathrm{O}_{2} \text { content. }\end{array}$ & [52] \\
\hline
\end{tabular}


Table 3. Cont.

\begin{tabular}{|c|c|c|c|c|}
\hline NPs & Plant & Pathogen & Summary Research & Reference \\
\hline $\begin{array}{l}\text { CS-NPs with DLS } \\
(126.2 \mathrm{~nm}, \text { PDI } 0.44 \\
\text { and } 27.8 \mathrm{mV})\end{array}$ & $\begin{array}{l}\text { Chilli } \\
\text { Papaya }\end{array}$ & $\begin{array}{l}\text { C. gloeosporioides } \\
\text { /Anthracnose }\end{array}$ & $\begin{array}{l}\text { In vitro: CS-NPs inhibited mycelial growth } \\
\text { (85.7\%) and spore germination (61.2\%). } \\
\text { In vivo: preventive (soaking onto CS-NPs } \\
2 \mathrm{~mL} / \mathrm{L} \text { for } 60 \text { min before inoculate), } \\
\text { curative (soaking onto spore suspension for } \\
15 \text { min following air-dried and soaking onto } \\
\text { CS-NPs for } 60 \text { min). } \\
\text { Inhibition rates of preventive and curative } \\
\text { treatment were } 87.5 \% \text { and } 75 \% \text { for chilli and } \\
50 \% \text { and } 10 \% \text { for papaya. }\end{array}$ & [56] \\
\hline $\begin{array}{l}\text { CS-NPs with } \\
\text { size } 100 \mathrm{~nm}\end{array}$ & Tomato & $\begin{array}{l}\text { C. gloeosporioides, } \\
\text { P. capsici, } \\
\text { S. sclerotiorum, } \\
\text { F. oxysporum, } \\
\text { G. fujikuori, } \\
\text { E. carotovora subsp. } \\
\text { carotovora, } \\
\text { X. campestris pv. } \\
\text { vesicatoria }\end{array}$ & 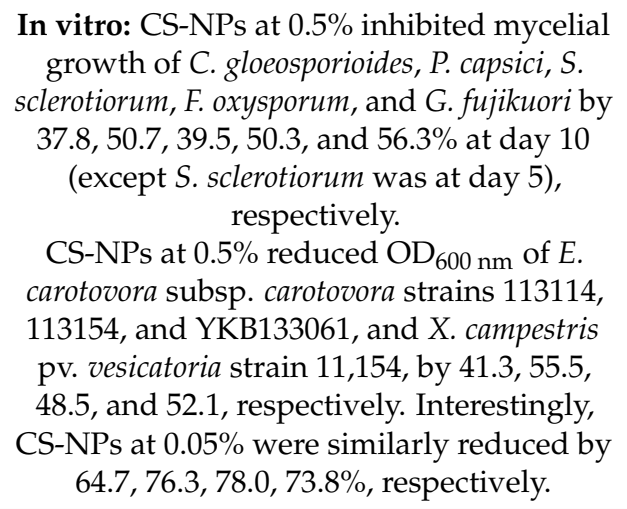 & [54] \\
\hline CS-NPs & Rice & $\begin{array}{c}\text { R. solani } \\
\text { /Sheath blight }\end{array}$ & $\begin{array}{c}\text { Detach leaves assay: Pre-treated CS-NPs } \\
\text { and CS at } 0.1 \% \text { reduced disease leaf area by } \\
92.78 \% \text { and } 78.89 \% \text {, respectively. } \\
\text { Greenhouse: Seed treat } 2 \mathrm{~h} \text {, soil amended } \\
\text { and foliar spraying } 15 \text { and } 30 \mathrm{DAP}, \\
\text { inoculate } 45 \mathrm{DAP} \\
\text { The disease was suppressed } 75.01 \% \text { and } \\
44.82 \% \text { when treated with CS-NPs and CS, } \\
\text { respectively. } \\
\text { Peroxidase, phenylalanine ammonia-lyase, } \\
\text { and chitinase activity were increased } 19-, \\
1.5-, \text { and } 1.9 \text {-fold, respectively. }\end{array}$ & [74] \\
\hline $\begin{array}{c}\text { CS-NPs with DLS } \\
(47 \mathrm{~nm}, \text { PDI } 0.45 \text {, and } \\
26.8 \mathrm{mV})\end{array}$ & $\begin{array}{l}\text { Tomato } \\
\text { Cereal }\end{array}$ & $\begin{array}{c}\text { C. michiganensis } \\
\text { /Bacterial canker } \\
\text { A. solani } \\
\text { /Leaf spot } \\
\text { F. graminearum } \\
\text { /Head blight and root } \\
\text { rot }\end{array}$ & $\begin{array}{l}\text { In vitro: lysis zone diameters of } \\
\text { C. michiganensis- and F. graminearum-treated } \\
\text { CS-NPs at } 0.014 \% \text { (in acetate buffer) were } \\
29.5 \text { and } 20 \mathrm{~mm} \text {, respectively. Similarly, CS } \\
\text { were } 22.5 \text { and } 18.0 \mathrm{~mm} \text {. } \\
\text { CS-NPs at } 0.03 \text { and } 0.04 \% \text { (in acetate buffer) } \\
\text { inhibited mycelial of } A \text {. solani by } 10 \text { and } 70 \% \\
\text { compared with CS, respectively. }\end{array}$ & {$[72]$} \\
\hline $\begin{array}{l}\text { CS-NPs with DLS } \\
\text { (192.5 nm, PDI 0.6, } \\
+45.33 \mathrm{mv}) \\
\text { CS-NPs-loaded } \\
\text { saponin with DLS } \\
\text { (373.9 nm (2 peaks), } \\
\text { PDI } 1.0,+31 \mathrm{mV}) \\
\text { And CS-NPs-loaded } \\
\text { copper }(\mathrm{Cu}) \text { with DLS } \\
\text { (196.4 nm, PDI 0.5, } \\
+88 \mathrm{mV})\end{array}$ & - & $\begin{array}{l}\text { A. alternata } \\
\text { M. phaseolina } \\
\text { R. solani }\end{array}$ & $\begin{array}{l}\text { In vitro: CS-NPs, CS-NPs-loaded saponin, } \\
\text { and CS-NPs-loaded copper at } 0.06-0.1 \% \\
\text { inhibited mycelial growth of } \text { A. alternate } \\
(80.1-82.2,78.3-80.9 \text {, and } 82.1-89.5 \%) \text { and } \\
R . \text { solani }(32.2-34.4,27.7 \text {, and } 62.5-63.0 \%) \\
\text { respectively. For M. phaseolina, } 3 \text { NPs at } 0.1 \% \\
\text { inhibited } 84,66.2 \text {, and } 60.1 \% \text {, respectively. } \\
\text { Moreover, } 3 \text { NPs at } 0.06-0.1 \% \text { inhibited } \\
\text { A. alternate spore germination by } 84.4-87.1 \text {, } \\
78.3-82.9 \text {, and } 83.3-87.4 \% \text {, respectively. }\end{array}$ & [51] \\
\hline
\end{tabular}


Table 3. Cont.

\begin{tabular}{|c|c|c|c|c|}
\hline NPs & Plant & Pathogen & Summary Research & Reference \\
\hline $\begin{array}{c}\text { CS-NPs-loaded copper } \\
(\mathrm{Cu}) \text { with DLS } \\
(374.3 \mathrm{~nm}, \text { PDI 0.33, } \\
\text { and } 22.6 \mathrm{mV}), \mathrm{TEM} \\
(150 \mathrm{~nm})\end{array}$ & Tomato & $\begin{array}{l}\text { A. solani } \\
\text { /Early blight } \\
\text { F. oxysporum } \\
\text { /Wilt }\end{array}$ & $\begin{array}{c}\text { Seeding: Treatment with CS-NPs-loaded } \\
\text { Cu at } 0.08,0.1, \text { and } 0.12 \% \text { increased seedling } \\
\text { vigor index }(33.9,33.7, \text { and } 24.3 \%) \text {, fresh } \\
\text { weight }(18.9,21.6 \text {, and } 16.2 \%) \text {, and dry } \\
\text { weight }(20.0,26.7, \text { and } 13.3 \%), \text { respectively. } \\
\text { In vitro: CS-NPs-loaded Cu at } 0.1 \% \\
\text { inhibited mycelium growth and spore } \\
\text { germination of } A \text {. solani and } F \text {. oxysporum by } \\
84.2 \% \text { and } 60.1 \% \text {, and } 73.3 \% \text { and } 79.9 \% \text {, } \\
\text { respectively. } \\
\text { Greenhouse: Spray at } 3-4 \text { DAI }(\text { A. solani }) ; \\
\text { apply to soil at } 3 \text { DAI (F. oxysporum). } \\
\text { NPs at } 0.1 \text { and } 0.12 \% \text { reduced early blight at } \\
84.2 \% \text { and } 87.7 \%, \text { fusarium wilt at } 49.9 \% \text {, } \\
\text { and } 61.1 \% \text {, respectively. }\end{array}$ & [75] \\
\hline
\end{tabular}

Greenhouse: Inoculate before planting by mix with soil, seed treat for $4,8 \mathrm{~h}$, and spray at 45 and 65 DAP.

CS-NPs-loaded copper $(\mathrm{Cu})$ with DLS

(295.4 nm, PDI 0.28, and $19.6 \mathrm{mV}$ )
F. verticillioides

/Post flowering stalk rot
Maize
NP treatment at $0.02-0.14 \%$ reduced disease severity $38.2-48.1 \%$ and $24.8-49.6 \%$ for seeds treated for 4 and $8 \mathrm{~h}$, respectively. Field: similar greenhouse experiments except inoculate at flowering stage.

NP treatment at $0.02-0.14 \%$ reduced disease severity by $23.5-33.9 \%$ and $2.55-15.8 \%$ for seed treated for 4 and $8 \mathrm{~h}$, respectively.

In vitro: $C S-N P s-l o a d e d ~ C u$ at 0.12 and $0.16 \%$ inhibited mycelial growth at 50.0 and $52.7 \%$, respectively.

Greenhouse: Treat seeds for $4 \mathrm{~h}$, foliar spray $35 \mathrm{DAP}$, and inoculate $45 \mathrm{DAP}$. Treatment with CS-NPs-loaded $\mathrm{Cu}$ at $0.01-0.08 \%$ increased plant height (15.9-47.0\%), stem diameter (82.9-102.9\%), root length (9.5-15.8\%), root number (20.9-46.3\%), and chlorophyll content (67.3-182.6\%). However, treatment at $0.16 \%$ reduced root length $(9.8 \%)$ and chlorophyll content (4.6-9.7\%), although the difference was non-significant. Moreover, treatment with NPs at 0.04-0.16 increased superoxide dismutases (1.8-2.2-fold), peroxidase (1.5-2.1-fold), phenylalanine ammonia-lyase (1.3-2.0-fold), and polyphenol oxidase (1.1-1.2-fold, which also reduced disease severity $43.86-48.48 \%$ ). Field: Treatment with CS-NPs-loaded $\mathrm{Cu}$ at $0.01-0.08 \%$ reduced disease severity by $27.72-28.53 \%$. Similarly, NPs at $0.12-0.16 \%$ reduced it by $30.42-33.88 \%$ and increased grain yield (25.4-29.3\%), 100 grain weight (14.4-16.9\%). 
Table 3. Cont.

\begin{tabular}{|c|c|c|c|c|}
\hline NPs & Plant & Pathogen & Summary Research & Reference \\
\hline $\begin{array}{c}\text { CS-NPs with DLS } \\
(86.8 \mathrm{~nm}, 32.4 \mathrm{mV}), \\
\text { CS-NPs load Harpin } \\
(\text { P. syringae pv. } \\
\text { syringae }), \text { and DLS } \\
(133.7 \mathrm{~nm}, 48.6 \mathrm{mV})\end{array}$ & Tomato & R. solani & $\begin{array}{l}\text { In planta: Treated amount of } 20 \mu \mathrm{g} \text { of CS-NPs } \\
\text { load Harpin enhanced cell death, necrotic } \\
\text { lesion, and } \mathrm{H}_{2} \mathrm{O}_{2} \text { accumulation faster and } \\
\text { stronger than Harpin protein only. Moreover, } \\
\text { treatment with NPs reduced fungal biomass } \\
\text { ( } 5 \text { folds), lesion diameter ( } 12 \text { folds), and failed } \\
\text { colonization in leaves, when compared with } \\
\text { control. For mechanism, peroxidase and } \\
\text { phenylalanine ammonia-lyase activity } \\
\text { steadily increased up to } 72 \mathrm{~h} \text {. The } \\
\text { transcriptome change, including defense } \\
\text { response, signal transduction, transport, } \\
\text { transcription, photosynthesis, housekeeping, } \\
\text { and aromatics biosynthesis, was enhanced } \\
\text { more than 2-fold at } 24,48, \text { and } 72 \mathrm{~h} \\
\text { after spraying. }\end{array}$ & {$[60]$} \\
\hline $\begin{array}{l}\text { CS-NPs } \\
50 \mathrm{~nm}\end{array}$ & Date palm & $\begin{array}{l}\text { F. oxysporum } \\
\text { /Vascular wilt }\end{array}$ & $\begin{array}{c}\text { Mix CS-NPs (ionic gelation method) and } \\
\text { Cu-NPs (chemical reduction method) to } \\
\text { obtain copper-chitosan } \\
\text { nanocomposition }(\mathrm{CuCs}) \\
\text { In vitro: CuCs at } 0.05-0.2 \% \text { could inhibit } \\
61.94-100 \% \text { mycelial growth. } \\
\text { Greenhouse: Apply } 50 \mathrm{~mL} \text { of CuCs to root } \\
\text { zone of seeding. } \\
\text { Treated CuCs increased plant } \\
\text { immunomodulatory, including total phenol } \\
\text { (1.1-1.5 folds), phenoloxidase (1.1-2.0 folds), } \\
\text { and peroxidase (1.6-3.0 folds), which led to } \\
\text { reduced disease by } 16.2-59.3 \% \text {. }\end{array}$ & [57] \\
\hline
\end{tabular}

In vitro: $\mathrm{CS}-\mathrm{NP}$ at $800 \mathrm{ppm}$ inhibited mycelial growth of $A$. tenuis, $A$. niger, $A$. terreus, $B$. bassiana, F. graminearum, $F$.

A. tenuis

A. niger

CS-NPs (DLS $180 \mathrm{~nm}$ with range $500-800 \mathrm{~nm}$ )

Ground nut oil seed
A. terreus

B. bassiana

F. graminearum

F. oxysporum S. rolfsii oxysporum, and S. rolfsii by $67.67,62.75,74.67$, $76.08,60.37,66.60$, and $37.41 \%$, respectively. Moreover, $0.2 \mathrm{~mL}$ of CS-NP at $125 \mathrm{ppm}$ inhibited F. graminearum by $44.3 \%$, higher than fungicide (8-hydroxy quinoline), which was $42.33 \%$. In addition, the CS-NP at $800 \mathrm{ppm}$ reduced zearalenone secreted by F. graminearum.

In vitro: NPs could inhibit mycelium growth at $47.7-65.2 \%$ and $0.08-0.16 \%$ and spore germination at $50.5-73.3 \%$ and $0.01-0.16 \%$. Greenhouse: Seed treat $4 \mathrm{~h}$, foliar spraying 35 DAP, inoculate 45 DAP.

CS-NPs-loaded zinc

(Zn) with DLS (387 $\mathrm{nm}$,

PDI 0.22, and $34 \mathrm{mV}$ ), TEM/SEM

(200-300 nm, spherical)
Maize
C. lunata

The superoxide dismutases, phenylalanine ammonia-lyase, polyphenol oxidase, $\mathrm{H}_{2} \mathrm{O}_{2}$ activity could be increased at 1.2-2.0, 2.0-3.0, 17.24-49.37, 1.5-2.6 folds when compared with the control, respectively. $\mathrm{H}_{2} \mathrm{O}_{2}$ and lignin localization were also increased. The DS was reduced $32.3-50.77 \%$. The plant height, stem diameter, root length were increased 30.3-60.3, 66.3-237.5, 2.7-61.1\%, respectively.

Field: The DS was reduced at $25.42-39.67 \%$. 
Table 3. Cont.

\begin{tabular}{|c|c|c|c|c|}
\hline NPs & Plant & Pathogen & Summary Research & Reference \\
\hline $\begin{array}{c}\text { CS-NPs-loaded SA } \\
\text { with DLS (368.7 nm, } \\
\text { PDI 0.1, and } 34.1 \mathrm{mV})\end{array}$ & Maize & $\begin{array}{l}\text { F. verticillioides } \\
\text { /Post-flowering } \\
\text { stalk rot }\end{array}$ & 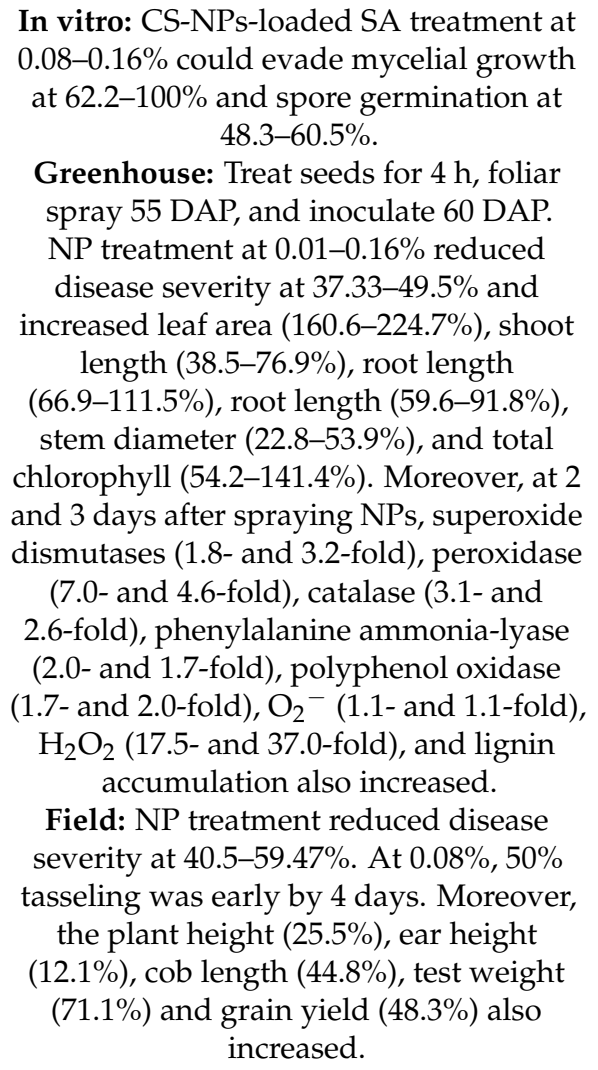 & [65] \\
\hline $\begin{array}{l}\text { CS-NPs with DLS } \\
\text { (bimodal particle with } \\
2.3 \text { and } 7.5 \mathrm{~nm} \text { ), TEM } \\
(1.5 \mathrm{~nm}), \text { and } \\
\text { CS-NPs-loaded } \\
\text { [H]hexaconazole, as } \\
\text { described in Table } 1\end{array}$ & Oil palm & G. boninense & $\begin{array}{c}\text { In vitro: } \mathrm{EC}_{50} \text { of hexaconazole, CS-NPs, } \\
\text { and four formulate CS-NPs-loaded } \\
\text { hexaconazole were } 21.4,1534.5,8.0 \text {, and } \\
18.4 \mathrm{ppb} \text {, respectively. Similar, fiducial } \\
\text { limit (lower-upper) was } 16.7-27.3, \\
\text { 494.0-13280.4, } \\
\text { 6.0-10.9 and 13.0-32.8 ppb, } \\
\text { respectively. }\end{array}$ & [58] \\
\hline $\begin{array}{l}\text { CS-NPs-loaded } \\
\text { Thiamine with DLS } \\
(596 \mathrm{~nm}, 37.7 \mathrm{mV}), \\
\text { HRTEM }(10-60 \mathrm{~nm})\end{array}$ & Chickpea & $\begin{array}{c}\text { F. oxysporum } \\
\text { /Wilt }\end{array}$ & $\begin{array}{c}\text { Seeding: soaking seed overnight at } 0.1 \% \\
\text { increased seedling vigor index by } 64.2 \% \\
\text { and Indole-3-acetic acid content } 10-\text { fold. } \\
\text { In vitro: CS-NPs-loaded thiamine did not } \\
\text { inhibit fungi even by } 0.1 \% \text {. } \\
\text { Greenhouse: Spraying } 12 \text { DAP and } \\
\text { inoculating } 15 \text { DAP } \\
\text { NP treatment at } 0.1 \% \text { reduced cell death } \\
\text { in } 2 \text { DAI compared with the control. } \\
\text { Moreover, shoot length, number of leaves } \\
\text { per plant, fresh weight, dry weight, and } \\
\text { number of secondary roots per plant also } \\
\text { were increased by } 15.3,14.4,37.7,20.0, \\
\text { and } 52.8 \% \text {, respectively. In leaves, } \\
\text { polyphenol oxidase, peroxidase, } \\
\beta-1,3 \text {-glucanase, chitinase, chitosanase, } \\
\text { and protease were increased by } 2.1-, 2.0-, \\
1.4-, 1.4-, 1.4-, \text { and } 1.1-\text { fold, respectively. In } \\
\text { root, this enzyme activity was increased } \\
2.0-, 1.3-, 1.1-, 1.3-, 1.3-, \text { and } 1.1-\text { fold, } \\
\text { respectively. }\end{array}$ & [66] \\
\hline
\end{tabular}


Table 3. Cont.

\begin{tabular}{|c|c|c|c|c|}
\hline NPs & Plant & Pathogen & Summary Research & Reference \\
\hline $\begin{array}{l}\text { CS-NPs-loaded copper } \\
(\mathrm{Cu}) \text { with DLS }(314 \mathrm{~nm} \text {, } \\
\text { PDI } 0.48 \text {, and } 19.5 \mathrm{mV})\end{array}$ & Soybean & $\begin{array}{c}\text { X. axonopodis pv. } \\
\text { glycine } \\
\text { /Bacterial pustule }\end{array}$ & $\begin{array}{l}\text { Greenhouse: Seed treatment for } 4 \mathrm{~h} \text {; } \\
\text { foliar spraying at trifoliate stage; and, } \\
\text { after disease occurrence, inoculate 35 DAP. } \\
\text { CS-NPs-loaded Cu at } 0.12-0.06 \% \text { reduced } \\
\text { disease by } 40.6-49.7 \% \text {. NP treatment at } \\
0.06 \% \text { increased plant height }(56.8 \%) \text {, root } \\
\text { length }(40.3 \%) \text {, and pod number }(7.2 \%) \text {. } \\
\text { NP treatment at } 0.02 \% \text { increased root } \\
\text { weight ( } 46.8 \%) \text {, nodule number ( } 44.2 \%) \text {, } \\
\text { and nodule weight }(125.8 \%) . \\
\text { Field: CS-NPs load Cu treatment at } 0.06 \% \\
\text { reduced disease by } 51.3 \% \text { and increased } \\
\text { root length (60.9\%), root weight }(46.8 \%) \text {, } \\
\text { and pod number }(29.7 \%) .\end{array}$ & [80] \\
\hline
\end{tabular}

Leaf disk assay: two NP formulations not caused phytotoxicity upto $800 \mathrm{ppm}$.

Greenhouse: Cassava stalk-soaking for

$5 \mathrm{~min}$, foliar spraying at 28 and $42 \mathrm{DAP}$, and inoculate $44 \mathrm{DAP}$ with density

CS-NP-loaded SA with DLS (89.86 nm, PDI 0.36 , and $22.27 \mathrm{mV}$ ), CS-NP-loaded silver (Ag) with DLS (249 nm, PDI 0.53, and $13.53 \mathrm{mV}$ )

Cassava $\quad \begin{array}{r}\text { A. alternata } \\ \text { /Leaf spot }\end{array}$

$10^{4}$ conidial per $\mathrm{mL}$ and $63 \mathrm{DAP}$ with density $10^{5}$ conidial per $\mathrm{mL}$.

CS-NP-loaded SA at $400 \mathrm{ppm}$ and

CS-NP-loaded Ag at 200, 400, and

$800 \mathrm{ppm}$ reduced disease by $68.9-73.6 \%$ at

56 DAP (first inoculate) and by $37.0-37.7 \%$

at 75 DAP (second inoculate). These

treatments increased the number of leaves

(45.1-82.4\%), the number of shoots

$(38.5-46.2 \%)$, the largest leaf area

(29.6-41.9\%), root length (11.6-29.9\%), and root weight (27.6-82.8\%).

Note: CS: chitosan; DAI: days after inoculate; DAP: days after planting; HRTEM: high-resolution transmission electron microscopy; NPs: nanoparticles; PDI: polydispersity index; SEM: scanning electron microscope; TEM: transmission electron microscopy.

\section{Conclusions and Future Perspectives}

Discovered since 1997, the studies on NPs synthesized by ionic gelation method have only received attention in the last ten years. The researches on using these NPs in plant disease management has only been interested in the last five years. With the advantage of being easy to implement, both CS-NP and CS-NP-loaded active ingredients $(\mathrm{Cu}$, Saponin, Harpin protein, Zn, SA, Hexaconazole, NPK, Thiamine, Silicon, and Ag) are effective in plant disease management and enhancing plant growth depending on the concentration and application method by direct or indirect mechanisms. CS-NP-loaded active ingredients constitute the "drug delivery system" model. The effectiveness of disease management and enhanced plant growth of CS-NP or CS-NP depend on the mechanism of CS (carrier) and active ingredients (drug). At higher concentrations, CS-NP or CS-NP-loaded active ingredients are effective in directly inhibiting phytopathogens. This can be applied to control when the disease has broken out. In addition, CS-NP and CS-NP-loaded active ingredients at lower concentrations can indirectly reduce disease through activation of plant's innate immunity, including stimulating cell death, $\mathrm{H}_{2} \mathrm{O}_{2}$ accumulation, oxidative burst $\left(\mathrm{O}_{2}{ }^{-}\right)$, enzymes ( $\beta-1,3$-glucanase, catalase, chitinase, chitosanase, peroxidase, phenoloxidases, phenoloxidases, phenylalanine ammonia-lyase, polyphenol oxidase, protease, and superoxide dismutases), and secondary metabolites (total phenols, lignin). Moreover, their treatment can enhance transcriptome changes, including defense response, signal transduction, transport, transcription, photosynthesis, housekeeping, and aromatic biosyn- 
thesis. In nature, plant diseases often have seasonal outbreaks. Periodical pre-treat CS-NP or CS-NP-loaded active ingredients at sensitive periods can prevent disease and reduce the consequences of disease outbreaks. Furthermore, CS-NP and CS-NP-loaded active ingredients can enhance indole-3-acetic acid, $\alpha$-amylase, protease, chlorophyll, carotenoid content, and photosynthesis rates, leading to increased plant growth, yield, and quality. When plants grow well, their health is enhanced and they can better tolerance diseases. In particular, CS-NP and CS-NP-loaded active ingredients are nano-sized, have a positive charge, and are able to easily penetrate cells or stick to plant surfaces. Moreover, the active ingredient can be slowly released into plant and easily absorbed with no waste. The CS (carrier) as a nitrogen source enhances cell division, cell elongation, enzymatic activation, and synthesis of protein. These preeminent characteristics lead to CS-NP or CS-NP-loaded active ingredients being more effective than CS or active ingredients alone. The CS-NP-loaded active ingredients are more interested in evaluating effectiveness in greenhouse and filed conditions. Most of the studies are more interested in fungal diseases (Alternaria spp., Aspergillus spp., B. bassiana, C. gloeosporioides, C. lunata, Fusarium spp., G. boninense, G. fujikuori, M. phaseolina, P. capsici, P. grisea, R. solani, S. sclerotiorum, and $S$. rolfsii) and bacteria (C. michiganensis, E. carotovora subsp. carotovora, and Xanthomonas spp.) than viruses, phytoplasma, viroid, and nematode. Many crops, including cassava, chickpea, chilli, date palm, fingermillet, maize, papaya, rice, soybean, tomato, and wheat, have been evaluated in in vivo or greenhouse conditions. However, field experiments are still limited as only maize (with CS-NP-loaded Cu, Zn, SA, and Silicon) and soybean (with CS-NP-loaded $\mathrm{Cu}$ ) have been evaluated for managing post-flowering stalk rot, Curvularia leaf spot, and bacterial pustule disease and/or enhancing plant growth.

Nanotechnology is the trend of the future. Easy access and dissemination of nano pesticides are essential, especially in developing areas. Since 2019, five of eight studies performed in field conditions have shown interest in CS-NPs synthesized by the ionic gelation method. In the future, new active ingredients could be loaded into CS-NPs or new polymers with anions by ionic gelation methods and used to improve crop yields. A hypothesis is proposed that "mixing CS and TPP under stirring conditions will lead to CS-NPs formation"; then, character or not, they will still be NPs and possess the superiority of NPs. Therefore, the "legendary" pairs of counter ions, CS and TPP, can be studied for immediate application in fields in developing regions where advanced research facilities are limited to building sustainable agriculture.

Author Contributions: Conceptualization: N.B., A.K. and T.L.T.; Data curation: W.T. and C.S.; Formal analysis: R.S. and C.S; Funding acquisition: N.B.; Investigation: N.K.P.; Methodology: N.H.H. and J.T.; Project administration: N.H.H. and T.L.T.; Resources: R.S. and W.T.; Software: N.K.P.; Supervision: N.B., A.K. and T.L.T.; Validation: N.B., A.K. and T.L.T.; Visualization: N.H.H.; Writing-original draft: N.H.H., T.L.T., W.T. and J.T.; Writing—review and editing: C.S., R.S., N.K.P., A.K. and N.B. All authors have read and agreed to the published version of the manuscript.

Funding: This research was funded by Suranaree University of Technology, grant number OROG Scholarship (M6201616) to Nguyen Huy Hoang. Moreover, this work was also supported by (i) Suranaree University of Technology; (ii) Thailand Science Research and Innovation (TSRI); and (iii) National Science, Research and Innovation Fund (NSRF) (project code 160342).

Institutional Review Board Statement: Not applicable.

Informed Consent Statement: Not applicable.

Data Availability Statement: Not applicable.

Acknowledgments: We would like to give special thanks to the School of Chemistry; Institute of Science; the Plant Pathology and Biopesticide Laboratory; School of Crop Production Technology; Institute of Agricultural Technology; and Suranaree University of Technology, Thailand for supporting our research project.

Conflicts of Interest: The authors declare no conflict of interest. 


\section{References}

1. Islam, S.M.F.; Karim, Z. World's demand for food and water: The consequences of climate change. In Desalination-Challenges and Opportunities; Farahani, M.H.D.A., Vatanpour, V., Taheri, A.H., Eds.; IntechOpen: London, UK, 2019; Chapter 4; pp. 57-84.

2. $\quad$ van Dijk, M.; Morley, T.; Rau, M.L.; Saghai, Y. A meta-analysis of projected global food demand and population at risk of hunger for the period 2010-2050. Nat. Food 2021, 2, 494-501. [CrossRef]

3. Moore, D.; Robson, G.D.; Trinci, A.P.J. 21st Century Guidebook to Fungi, 1st ed.; Cambridge University Press: Cambridge, UK, 2011; pp. 367-391.

4. Khan, M.R.; Sharma, R.K. Fusarium-nematode wilt disease complexes, etiology and mechanism of development. Indian Phytopathol. 2020, 73, 615-628. [CrossRef]

5. Savary, S.; Ficke, A.; Aubertot, J.N.; Hollier, C. Crop losses due to diseases and their implications for global food production losses and food security. Food Sec. 2012, 4, 519-537. [CrossRef]

6. Godfray, H.C.J.; Mason-D'Croz, D.; Robinson, S. Food system consequences of a fungal disease epidemic in a major crop. Philos. Trans. R. Soc. Lond. B Biol. Sci. 2016, 371, 20150467. [CrossRef]

7. Schreinemachers, P.; Chen, H.P.; Nguyen, T.T.L.; Buntong, B.; Bouapao, L.; Gautam, S.; Le, N.T.; Pinn, T.; Vilaysone, P.; Srinivasan, R. Too much to handle? Pesticide dependence of smallholder vegetable farmers in Southeast Asia. Sci. Total Environ. 2017, 593, 470-477. [CrossRef]

8. Pimentel, D. Amounts of pesticides reaching target pests: Environmental impacts and ethics. J. Agric. Environ. Ethics 1995, 8 , 17-29. [CrossRef]

9. Matthews, G.A. Pesticides: Health, Safety and the Environment, 1st ed.; Blackwell Publishing Ltd.: Oxford, UK, 2006 ; pp. 168-183.

10. Piesse, M. Global Food and Water Security in 2050: Demographic Change and Increased Demand. Strategic Analysis Paper. 2020. Available online: https:/ / www.futuredirections.org.au/wp-content/uploads/2020/02/Global-Food-and-Water-Security-in2050-Demographic-Change-and-Increased-Demand.pdf (accessed on 13 January 2022).

11. Duhan, J.S.; Kumar, R.; Kumar, N.; Kaur, P.; Nehra, K.; Duhan, S. Nanotechnology: The new perspective in precision agriculture. Biotechnol. Rep. 2017, 15, 11-23. [CrossRef]

12. Sanzari, I.; Leone, A.; Ambrosone, A. Nanotechnology in plant science: To make a long story short. Front. Bioeng. Biotechnol. 2019, 7, 120. [CrossRef]

13. Shang, Y.; Hasan, M.; Ahammed, G.J.; Li, M.; Yin, H.; Zhou, J. Applications of nanotechnology in plant growth and crop protection: A review. Molecules 2019, 24, 2558. [CrossRef]

14. Paramo, L.A.; Feregrino-Pérez, A.A.; Guevara, R.; Mendoza, S.; Esquivel, K. Nanoparticles in agroindustry: Applications, toxicity, challenges, and trends. Nanomaterials 2020, 10, 1654. [CrossRef]

15. Liu, C.; Zhou, H.; Zhou, J. The Applications of Nanotechnology in Crop Production. Molecules 2021, 26, 7070. [CrossRef] [PubMed]

16. Rajput, V.D.; Singh, A.; Minkina, T.; Rawat, S.; Mandzhieva, S.; Sushkova, S.; Shuvaeva, V.; Nazarenko, O.; Rajput, P.; Verma, K.K.; et al. Nano-Enabled Products: Challenges and Opportunities for Sustainable Agriculture. Plants 2021, $10,2727$. [CrossRef] [PubMed]

17. Hassanisaadi, M.; Barani, M.; Rahdar, A.; Heidary, M.; Thysiadou, A.; Kyzas, G.Z. Role of agrochemical-based nanomaterials in plants: Biotic and abiotic stress with germination improvement of seeds. Plant Growth Regul. 2022, 1-44. [CrossRef]

18. Kashyap, P.L.; Xiang, X.; Heiden, P. Chitosan nanoparticle based delivery systems for sustainable agriculture. Int. J. Biol. Macromol. 2015, 77, 36-51. [CrossRef] [PubMed]

19. Das, S.S.; Bharadwaj, P.; Bilal, M.; Barani, M.; Rahdar, A.; Taboada, P.; Bungau, S.; Kyzas, G.Z. Stimuli-Responsive Polymeric Nanocarriers for Drug Delivery, Imaging, and Theragnosis. Polymers 2020, 12, 1397. [CrossRef] [PubMed]

20. Amiri, M.S.; Mohammadzadeh, V.; Yazdi, M.E.T.; Barani, M.; Rahdar, A.; Kyzas, G.Z. Plant-Based Gums and Mucilages Applications in Pharmacology and Nanomedicine: A Review. Molecules 2021, 26, 1770. [CrossRef]

21. Barani, M.; Sangiovanni, E.; Angarano, M.; Rajizadeh, M.A.; Mehrabani, M.; Piazza, S.; Gangadharappa, H.V.; Pardakhty, A.; Mehrbani, M.; Dell'Agli, M.; et al. Phytosomes as innovative delivery systems for phytochemicals: A comprehensive review of literature. Int. J. Nanomed. 2021, 16, 6983. [CrossRef]

22. Soltani, M.D.; Meftahizadeh, H.; Barani, M.; Rahdar, A.; Hosseinikhah, S.M.; Hatami, M.; Ghorbanpour, M. Guar (Cyamopsis tetragonoloba L.) plant gum: From biological applications to advanced nanomedicine. Int. J. Biol. Macromol. 2021, 193, 1972-1985. [CrossRef]

23. Worrall, E.A.; Hamid, A.; Mody, K.T.; Mitter, N.; Pappu, H.R. Nanotechnology for plant disease management. Agronomy 2018, 8, 285. [CrossRef]

24. Elmer, W.; White, J.C. The future of nanotechnology in plant pathology. Annu. Rev. Phytopathol. 2018, 56, 111-133. [CrossRef]

25. Mittal, D.; Kaur, G.; Singh, P.; Yadav, K.; Ali, S.A. Nanoparticle-based sustainable agriculture and food science: Recent advances and future outlook. Front. Nanotechnol. 2020, 2, 10. [CrossRef]

26. Servin, A.; Elmer, W.; Mukherjee, A.; De la Torre-Roche, R.; Hamdi, H.; White, J.C.; Bindraban, P.; Dimkpa, C. A review of the use of engineered nanomaterials to suppress plant disease and enhance crop yield. J. Nanopart Res. 2015, 17, 92. [CrossRef]

27. Okey-Onyesolu, C.F.; Hassanisaadi, M.; Bilal, M.; Barani, M.; Rahdar, A.; Iqbal, J.; Kyzas, G.Z. Nanomaterials as nanofertilizers and nanopesticides: An overview. ChemistrySelect 2021, 6, 8645-8663. [CrossRef]

28. Singh, J.; Dutta, T.; Kim, K.H.; Rawat, M.; Samddar, P.; Kumar, P. 'Green'synthesis of metals and their oxide nanoparticles: Applications for environmental remediation. J. Nanobiotechnol. 2018, 16, 1-24. [CrossRef] [PubMed] 
29. Goutam, S.P.; Saxena, G.; Roy, D.; Yadav, A.K.; Bharagava, R.N. Green Synthesis of Nanoparticles and Their Applications in Water and Wastewater Treatment. In Bioremediation of Industrial Waste for Environmental Safety; SaxenaRam, G., Bharagava, N., Eds.; Springer: Singapore, 2020; Chapter 16; pp. 349-379.

30. Calvo, P.; Remunan-Lopez, C.; Vila-Jato, J.L.; Alonso, M.J. Novel hydrophilic chitosan-polyethylene oxide nanoparticles as protein carriers. J. Appl. Polym. Sci. 1997, 63, 125-132. [CrossRef]

31. Calvo, P.; Remuñan-López, C.; Vila-Jato, J.L.; Alonso, M.J. Chitosan and chitosan/ethylene oxide-propylene oxide block copolymer nanoparticles as novel carriers for proteins and vaccines. Pharm. Res. 1997, 14, 1431-1436. [CrossRef] [PubMed]

32. Du, W.L.; Niu, S.S.; Xu, Y.L.; Xu, Z.R.; Fan, C.L. Antibacterial activity of chitosan tripolyphosphate nanoparticles loaded with various metal ions. Carbohydr. Polym. 2009, 75, 385-389. [CrossRef]

33. Debnath, S.; Kumar, R.S.; Babu, M.N. Ionotropic gelation-a novel method to prepare chitosan nanoparticles. Res. J. Pharm. Tech. 2011, 4, 492-495.

34. Koukaras, E.N.; Papadimitriou, S.A.; Bikiaris, D.N.; Froudakis, G.E. Insight on the formation of chitosan nanoparticles through ionotropic gelation with tripolyphosphate. Mol. Pharm. 2012, 9, 2856-2862. [CrossRef]

35. Kunjachan, S.; Jose, S.; Lammers, T. Understanding the mechanism of ionic gelation for synthesis of chitosan nanoparticles using qualitative techniques. Asian J. Pharm. 2014, 4, 148-153. [CrossRef]

36. Giri, T.K. Alginate containing nanoarchitectonics for improved cancer therapy. In Nanoarchitectonics for Smart Delivery and Drug Targeting; Holban, A.M., Grumezescu, A.M., Eds.; William Andrew: Norwich, NY, USA, 2016; Chapter 20; pp. 565-588.

37. Pedroso-Santana, S.; Fleitas-Salazar, N. Ionotropic gelation method in the synthesis of nanoparticles/microparticles for biomedical purposes. Polym. Int. 2020, 69, 443-447. [CrossRef]

38. Lima, D.D.S.; Gullon, B.; Cardelle-Cobas, A.; Brito, L.M.; Rodrigues, K.A.; Quelemes, P.V.; Ramos-Jesus, J.; Arcanjo, D.D.; Plácido, A.; Batziou, K.; et al. Chitosan-based silver nanoparticles: A study of the antibacterial, antileishmanial and cytotoxic effects. J. Bioact. Compat. Polym. 2017, 32, 397-410. [CrossRef]

39. Google Schoolar. Available online: https://scholar.google.com/scholar?q=\%E2\%80\%9Cionic+gelation $\% \mathrm{E} 2 \% 80 \% 9 \mathrm{D}+\% 2 \mathrm{~B}+\% 2$ 2nanoparticles\%22\&hl=en\&as_sdt=0,5\&as_vis=1 (accessed on 13 January 2022).

40. Racoviţă, S.; Vasiliu, S.; Popa, M.; Luca, C. Polysaccharides based on micro-and nanoparticles obtained by ionic gelation and their applications as drug delivery systems. Rev. Roum. Chim. 2009, 54, 709-718.

41. Mudgil, M.; Gupta, N.; Nagpal, M.; Pawar, P. Nanotechnology: A new approach for ocular drug delivery system. Int. J. Pharm. Pharm. Sci. 2012, 4, 105-112.

42. Malerba, M.; Cerana, R. Recent applications of chitin-and chitosan-based polymers in plants. Polymers 2019, 11, 839. [CrossRef] [PubMed]

43. Chakraborty, M.; Hasanuzzaman, M.; Rahman, M.; Khan, M.; Rahman, A.; Bhowmik, P.; Mahmud, N.U.; Tanveer, M.; Islam, T. Mechanism of plant growth promotion and disease suppression by chitosan biopolymer. Agriculture 2020, 10, 624. [CrossRef]

44. Food and Agriculture Organization of the United Nations. The State of World Fisheries and Aquaculture. Available online: https:/ / www.fao.org/3/i3720e/i3720e.pdf (accessed on 13 January 2022).

45. Food and Agriculture Organization of the United Nations (FAO). The State of World Fisheries and Aquaculture 2020. Sustainability in Action. 2020. Available online: https:/ / www.fao.org/3/ca9229en/ca9229en.pdf (accessed on 13 January 2022).

46. Agarwal, M.; Nagar, D.P.; Srivastava, N.; Agarwal, M.K. Chitosan nanoparticles-based drug delivery: An update. Int. J. Adv. Multidiscip. Res. 2015, 2,1-13.

47. U.S. Food and Drug Administration, Title 21-Food and drugs. Chapter I-Food and Drug Administration. Subchapter B-Food for Human Consumption. Part 182-Substances Generally Recognized as Safe. 2021. Available online: https:/ / www.accessdata.fda. gov/scripts/cdrh/cfdocs/cfcfr/CFRSearch.cfm?fr=182.6810 (accessed on 13 January 2022).

48. Wang, Y.; Li, P.; Truong-Dinh Tran, T.; Zhang, J.; Kong, L. Manufacturing techniques and surface engineering of polymer based nanoparticles for targeted drug delivery to cancer. Nanomaterials 2016, 6, 26. [CrossRef]

49. Maluin, F.N.; Hussein, M.Z. Chitosan-based agronanochemicals as a sustainable alternative in crop protection. Molecules 2020, 25, 1611. [CrossRef]

50. Devi, K.A.; Prajapati, D.; Kumar, A.; Pal, A.; Bhagat, D.; Singh, B.R.; Adholeya, A.; Saharan, V. Smart Nano-Chitosan for Fungal Disease Control. In Nanopesticides; Fraceto, L.F., de Castro, V.L.S.S., Grillo, R., Ávila., D., Oliveira, H.C., Lima, R., Eds.; Springer: Cham, Switzerland, 2020; Chapter 2; pp. 23-47.

51. Saharan, V.; Mehrotra, A.; Khatik, R.; Rawal, P.; Sharma, S.S.; Pal, A. Synthesis of chitosan based nanoparticles and their in vitro evaluation against phytopathogenic fungi. Int. J. Biol. Macromol. 2013, 62, 677-683. [CrossRef]

52. Kheiri, A.; Jorf, S.M.; Malihipour, A.; Saremi, H.; Nikkhah, M. Synthesis and characterization of chitosan nanoparticles and their effect on Fusarium head blight and oxidative activity in wheat. Int. J. Biol. Macromol. 2017, 102, 526-538. [CrossRef] [PubMed]

53. Bangun, H.; Tandiono, S.; Arianto, A. Preparation and evaluation of chitosan-tripolyphosphate nanoparticles suspension as an antibacterial agent. J. Appl. Pharm. Sci. 2018, 8, 147-156.

54. Oh, J.W.; Chun, S.C.; Chandrasekaran, M. Preparation and in vitro characterization of chitosan nanoparticles and their broadspectrum antifungal action compared to antibacterial activities against phytopathogens of tomato. Agronomy 2019, 9, 21. [CrossRef]

55. Rodriguez, V.A.; Bolla, P.K.; Kalhapure, R.S.; Boddu, S.H.S.; Neupane, R.; Franco, J.; Renukuntla, J. Preparation and Characterization of Furosemide-Silver Complex Loaded Chitosan Nanoparticles. Processes 2019, 7, 206. [CrossRef] 
56. Suryadi, Y.; Priyatno, T.P.; Samudra, I.; Susilowati, D.N.; Sriharyani, T.S.; Syaefudin, S. Control of anthracnose disease (Colletotrichum gloeosporioides) using nano chitosan hydrolyzed by chitinase derived from Burkholderia cepacia Isolate E76. J. AgroBiogen 2019, 13, 111-122. [CrossRef]

57. Mohamed, E.A.; Gaber, M.H.; Elsharabasy, S.F. Evaluating the in vivo efficacy of copper-chitosan nanocomposition for treating vascular wilt disease in date palm. Int. J. Agric. Environ. Biotechnol. 2018, 3, 239085. [CrossRef]

58. Maluin, F.N.; Hussein, M.Z.; Yusof, N.A.; Fakurazi, S.; Idris, A.S.; Zainol Hilmi, N.H.; Jeffery Daim, L.D. Preparation of chitosanhexaconazole nanoparticles as fungicide nanodelivery system for combating Ganoderma disease in oil palm. Molecules 2019, 24, 2498. [CrossRef]

59. Manikandan, A.; Sathiyabama, M. Preparation of chitosan nanoparticles and its effect on detached rice leaves infected with Pyricularia grisea. Int. J. Biol. Macromol. 2016, 84, 58-61. [CrossRef]

60. Nadendla, S.R.; Rani, T.S.; Vaikuntapu, P.R.; Maddu, R.R.; Podile, A.R. Harpin Pss encapsulation in chitosan nanoparticles for improved bioavailability and disease resistance in tomato. Carbohydr. Polym. 2018, 199, 11-19. [CrossRef]

61. Clayton, K.N.; Salameh, J.W.; Wereley, S.T.; Kinzer-Ursem, T.L. Physical characterization of nanoparticle size and surface modification using particle scattering diffusometry. Biomicrofluidics 2016, 10, 054107. [CrossRef]

62. Selvamani, V. Stability studies on nanomaterials used in drugs. In Characterization and Biology of Nanomaterials for Drug Delivery; Mohapatra, S., Ranjan, S., Dasgupta, N., Mishra, R., Thomas, S., Eds.; Elsevier: Amsterdam, The Netherlands, 2019; Chapter 15; pp. $425-444$.

63. Sathiyabama, M.; Parthasarathy, R. Biological preparation of chitosan nanoparticles and its in vitro antifungal efficacy against some phytopathogenic fungi. Carbohydr. Polym. 2016, 151, 321-325. [CrossRef] [PubMed]

64. Ji, J.; Hao, S.; Wu, D.; Huang, R.; Xu, Y. Preparation, characterization and in vitro release of chitosan nanoparticles loaded with gentamicin and salicylic acid. Carbohydr. Polym. 2011, 85, 803-808. [CrossRef]

65. Kumaraswamy, R.V.; Kumari, S.; Choudhary, R.C.; Sharma, S.S.; Pal, A.; Raliya, R.; Biswas, P.; Saharan, V. Salicylic acid functionalized chitosan nanoparticle: A sustainable biostimulant for plant. Int. J. Biol. Macromol. 2019, 123, 59-69. [CrossRef] [PubMed]

66. Muthukrishnan, S.; Murugan, I.; Selvaraj, M. Chitosan nanoparticles loaded with thiamine stimulate growth and enhances protection against wilt disease in Chickpea. Carbohydr. Polym. 2019, 212, 169-177. [CrossRef]

67. Kain, D.; Kumar, S. Synthesis and characterization of chitosan nanoparticles of Achillea millefolium L. and their activities. F1000Research 2020, 9, 1297. [CrossRef]

68. Bocchetta, P. Ionotropic gelation of chitosan for next-generation composite proton conducting flat structures. Molecules 2020, 25, 1632. [CrossRef]

69. Hoang, N.H.; Le Thanh, T.; Thepbandit, W.; Treekoon, J.; Saengchan, C.; Sangpueak, R.; Papathoti, N.K.; Kamkaew, A.; Buensanteai, N. Efficacy of chitosan nanoparticle loaded-salicylic acid and -silver on management cassava leaf spot disease. Polymers 2022. accepted.

70. Das, S.; Pattanayak, S. Nanotechnological Approaches in Sustainable Agriculture and Plant Disease Management. In Organic Agriculture; Das, S.K., Ed.; IntechOpen: London, UK, 2020; Chapter 2; pp. 13-30.

71. Sathiyabama, M.; Manikandan, A. Chitosan nanoparticle induced defense responses in fingermillet plants against blast disease caused by Pyricularia grisea (Cke). Sacc. Carbohydr. Polym. 2016, 154, 241-246. [CrossRef]

72. Popova, E.V.; Zorin, I.M.; Domnina, N.S.; Novikova, I.I.; Krasnobaeva, I.L. Chitosan-Tripolyphosphate Nanoparticles: Synthesis by the Ionic Gelation Method, Properties, and Biological Activity. Russ. J. Gen. Chem. 2020, 90, 1304-1311. [CrossRef]

73. Abdel-Aliem, H.A.; Gibriel, A.Y.; Rasmy, N.M.; Sahab, A.F.; El-Nekeety, A.A.; Abdel-Wahhab, M.A. Antifungal efficacy of chitosan nanoparticles against phytopathogenic fungi and inhibition of zearalenone production by Fusarium graminearum. Comun. Sci. 2019, 10, 338-345. [CrossRef]

74. Divya, K.; Thampi, M.; Vijayan, S.; Varghese, S.; Jisha, M.S. Induction of defence response in Oryza sativa L. against Rhizoctonia solani (Kuhn) by chitosan nanoparticles. Microb. Pathog. 2020, 149, 104525. [CrossRef] [PubMed]

75. Choudhary, R.C.; Kumaraswamy, R.V.; Kumari, S.; Sharma, S.S.; Pal, A.; Raliya, R.; Biswas, P.; Saharan, V. Cu-chitosan nanoparticle boost defense responses and plant growth in maize (Zea mays L.). Sci. Rep. 2017, 7, 9754. [CrossRef]

76. Saharan, V.; Sharma, G.; Yadav, M.; Choudhary, M.K.; Sharma, S.S.; Pal, A.; Raliya, R.; Biswas, P. Synthesis and in vitro antifungal efficacy of $\mathrm{Cu}$-chitosan nanoparticles against pathogenic fungi of tomato. Int. J. Biol. Macromol. 2015, 75, 346-353. [CrossRef]

77. Choudhary, R.C.; Kumaraswamy, R.V.; Kumari, S.; Sharma, S.S.; Pal, A.; Raliya, R.; Biswas, P.; Saharan, V. Zinc encapsulated chitosan nanoparticle to promote maize crop yield. Int. J. Biol. Macromol. 2019, 127, 126-135. [CrossRef] [PubMed]

78. Choudhary, M.K.; Joshi, A.; Sharma, S.S.; Saharan, V. Effect of laboratory synthesized Cu-Chitosan nanocomposites on control of PFSR disease of Maize caused by Fusarium verticillioids. Int. J. Curr. Microbiol. Appl. Sci. 2017, 6, 1656-1664. [CrossRef]

79. Dong, H.; Delaney, T.P.; Bauer, D.W.; Beer, S.V. Harpin induces disease resistance in Arabidopsis through the systemic acquired resistance pathway mediated by salicylic acid and the NIM1 gene. Plant J. 1999, 20, 207-215. [CrossRef] [PubMed]

80. Swati, K.J.A.J. Cu-chitosan nanoparticle induced plant growth and antibacterial activity against bacterial pustule disease in soybean [Glycine max (L.)]. J. Pharmacogn. Phytochem. 2020, 9, 450-455.

81. Elemike, E.E.; Uzoh, I.M.; Onwudiwe, D.C.; Babalola, O.O. The role of nanotechnology in the fortification of plant nutrients and improvement of crop production. Appl. Sci. 2019, 9, 499. [CrossRef] 
82. Ashraf, S.A.; Siddiqui, A.J.; Abd Elmoneim, O.E.; Khan, M.I.; Patel, M.; Alreshidi, M.; Moin, A.; Singh, R.; Snoussi, M.; Adnan, M. Innovations in nanoscience for the sustainable development of food and agriculture with implications on health and environment. Sci. Total Environ. 2021, 768, 144990. [CrossRef]

83. Ur Rahim, H.; Qaswar, M.; Uddin, M.; Giannini, C.; Herrera, M.L.; Rea, G. Nano-enable materials promoting sustainability and resilience in modern agriculture. Nanomaterials 2021, 11, 2068. [CrossRef]

84. Zielińska, A.; Costa, B.; Ferreira, M.V.; Miguéis, D.; Louros, J.; Durazzo, A.; Lucarini, M.; Eder, P.; Chaud, M.V.; Morsink, M.; et al. Nanotoxicology and nanosafety: Safety-by-design and testing at glance. Int. J. Environ. Res. Public Health 2020, $17,4657$. [CrossRef] [PubMed]

85. Choudhary, R.C.; Joshi, A.; Kumari, S.; Kumaraswamy, R.V.; Saharan, V. Preparation of Cu-chitosan nanoparticle and its effect on growth and enzyme activity during seed germination in maize. J. Pharmacogn. Phytochem. 2017, 6, 669-673.

86. Ha, N.M.C.; Nguyen, T.H.; Wang, S.L.; Nguyen, A.D. Preparation of NPK nanofertilizer based on chitosan nanoparticles and its effect on biophysical characteristics and growth of coffee in green house. Res. Chem. Intermed. 2019, 45, 51-63. [CrossRef]

87. Kumaraswamy, R.V.; Saharan, V.; Kumari, S.; Choudhary, R.C.; Pal, A.; Sharma, S.S.; Rakshit, S.; Raliya, R.; Biswas, P. Chitosansilicon nanofertilizer to enhance plant growth and yield in maize (Zea mays L.). Plant Physiol. Biochem. 2021, 159, 53-66. [CrossRef] 